\title{
Response-adaptive randomization: an overview of designs and asymptotic theory
}

\author{
Li-XiN ZHANG \\ Department of Mathematics, Zhejiang University, Hangzhou 310027 \\ and Zhejiang University City College \\ Email:stazlx@zju.edu.cn
}

\section{Introduction}

In clinical trials, patients normally arrive sequentially. Response-adaptive treatment allocation procedures are sequentially adaptive schemes that uses past treatment assignments and patients' responses to select future treatment assignments. Historically, response-adaptive treatment allocation procedures were developed for the purpose of assigning more patients to the empirically better treatment. Early important work on response-adaptive designs can be traced back to Thompson (1933) and Robbins (1952). Since then, a lot of responseadaptive designs have been proposed in literature. A history of the subject is discussed in Rosenberger and Lachin (2002) and $\mathrm{Hu}$ and Rosenberger (2006). The most famous nonrandomized response-adaptive treatment allocation procedure is the play-the-winner (PW) rule proposed by Zelen (1969), in which a success on a treatment results in the next patient's assignment to the same treatment, and a failure on the treatment results in the next patient's assignment to the opposite treatment. The idea of incorporating randomization into response-adaptive treatment assignments is due to Wei and Durham (1978), who proposed a randomized play-the-winner (RPW) rule for binary outcome trials. With this approach, each patient's treatment assignment is determined by drawing a ball from an urn, and the urn composition is updated by adding an additional ball of the same type if the patient's response is a success and an additional ball of the opposite type if the patient's response is a failure, so that the ball corresponding to the better treatment has a larger probability to be drawn. The RPW rule was used to design a pediatric trial of extracorporeal membrane oxygenation (ECMO; Bartlett et al., 1985), which compared the ECMO therapy

\footnotetext{
${ }^{1}$ Research supported by Grants from the National Natural Science Foundation of China (No. 11225104) and the Fundamental Research Funds for the Central Universities.
} 
versus the conventional therapy. Unfortunately, the trial provided very little information about survival rates of the two treatments. The trial stopped after enrolling 12 infants, of whom one infant was randomized to the conventional therapy and died and 11 infants were randomized to ECMO treatment and all survived. This design and subsequent data analysis generated a lot of controversy and much of the criticism of response-adaptive randomization (RAR) designs; in fact the ECMO trial example is still often used as a reason not to perform response-adaptive randomization at all. According to the modern theory on response-adaptive randomization designs, the failure of the ECMO trial can be explained mainly by the trial's small sample size and the poor operating characteristics of the RPW rule, in particular, the rule's high variability and dependence on the initial composition of the balls in the urn. Since the time of the ECMO trial, the body of knowledge on responseadaptive randomization has grown significantly and many new methods have been proposed to address past criticisms and concerns that have hindered the use of adaptive randomization in clinical trials. In this paper we provide an overview of important research works on response-adaptive randomization completed in the past decades. In the next section, we give the general framework. The RPW rule is only one example of a broad class of randomized urn models and is generalized to the "generalized Pólya urn model" (GPU). Other notable response-adaptive randomization urn designs with desirable statistical properties are proposed in the past decades including the "drop-the-loser (DL) rule" by Ivanova (2003), "sequential estimated-adjusted urn" (SEU) by Zhang, Hu and Cheung (2006), the "generalized drop-the-loser (GDL) rule" by Zhang et al. (2007), and the "immigrated urn (IMU) model" by Zhang et al. (2011). We state the theory on urn models in Section 3 , Modern research on response-adaptive randomization has focused on the development of optimal response-adaptive randomization procedures that maintain or increase power over traditional balanced randomization designs and minimize expected treatment failures. The optimization problem and the efficiency of the response-adaptive randomization designs are discussed in Section 4, where the methods and theoretical principles are proposed for defining a design with desirable or most desirable statistical properties. The asymptotic properties of several modern designs such as "doubly adaptive biased coin design" (DBCD) of Hu and Zhang (2004a), "efficient response adaptive randomization designs" (ERADE) of Hu, Zhang and $\mathrm{He}(2009)$ etc are presented. In section 5 , the selection bias and the lack of randomness 
are discussed. The measures for evaluating the selection bias and the degree of the lack of randomness are defined and the measure values of several randomization procedures are given. Survival and delayed responses are briefly discuss in Section 6, A theorem is given for showing that the mild delay will not effect the theoretical results of response-adaptive randomization designs. The last section gives a concluding remark.

\section{Framework}

We consider $K$-treatment clinical trials, $K \geq 2$. Suppose that the outcome of treatment $k$ follows a probability distribution $f_{k}\left(x \mid \boldsymbol{\theta}_{k}\right)$ indexed by a parameter $\boldsymbol{\theta}_{k}$. The patients arrive at the clinical trial sequentially and will be allocated to one of the $K$ treatments with a certain probability one by one. After the first $m$ assignments, the $(m+1)$-th patient will be assigned to treatment $k$ with a probability

$$
p_{m+1, k}, \quad k=1, \ldots, K
$$

The probabilities $p_{m+1, k} \mathrm{~s}$ are usually functions of the allocation results and outcomes of the treatments of previous $m$ trials. Let $N_{m, k}$ be the number of the patients assigned to the treatment $k$ in the first $m$ stages, $k=1, \ldots, K$, and $\boldsymbol{N}_{m}=\left(N_{m, 1}, \ldots, N_{m, K}\right)$. Two problems are always concerned in the adaptive randomization studies. One is the way for defining the allocation probabilities $p_{m+1, k} \mathrm{~s}$ so that the design will achieve the desired purposes in clinical trials. The other is the theory on the behaviors of $N_{m, k} \mathrm{~s}$ and related statistical inference.

When $K=2$, in the play-the-winner rule of Zelen (1969), the allocation probability $p_{m+1, k}$ is defined to be 1 if the $m$-th patient is assigned to treatment $k$ and success, and 0 otherwise, $k=1,2$. And in the randomized play-the-winner rule of Wei and Durham (1978), the allocation probability $p_{m+1, k}$ is defined to be the current proportion of balls of type $k$ in the urn:

$$
p_{m+1, k}=\frac{Y_{m, k}}{Y_{m, 1}+Y_{m, 2}}
$$

where $Y_{m, k}$ is the number of balls of type $k$ and is defined successively by $Y_{m, k}=Y_{m-1, k}+1$ if the $(m-1)$-th patient is assigned to treatment $k$ and successes, or the $(m-1)$-th patient is assigned to the other treatment and fails. For both the play-the-winner rule and randomized 
play-the-winner rule, it is shown that

$$
\frac{N_{n, k}}{n} \rightarrow \frac{q_{k}}{q_{1}+q_{2}} \text { in probability, }
$$

where $q_{k}$ is the failure rate of the treatment $k, k=1,2$.

\section{$3 \quad$ Urn Models}

In the latter part of the 20th century, most research on response-adaptive randomization focused on ad hoc designs based on stochastic processes such as urn models (see Rosenberger, 2002). Many of these were extensions of the randomized play-the-winner methodology, and they focused on putting more patients on the superior treatment. One large family is the family of the generalized Pólya urn models.

\subsection{Generalized Pólya Urn}

Consider an urn containing balls of $K$ types. At the beginning, the urn contains $\boldsymbol{Y}_{0}=$ $\left(Y_{0,1}, \ldots, Y_{0, K}\right)$ particles, where $Y_{0, k}>0$ denotes the number of particles of type $k, k=$ $1, \ldots, K$. After $m$ assignments, the urn composition is denoted by the row vector $\boldsymbol{Y}_{m}=$ $\left(Y_{m, 1}, \ldots, Y_{m, K}\right)$. The $(m+1)$-th patient is randomized to treatments by drawing a ball from the urn with replacement. If the ball drawn is of type $k$, then the patient is assigned to treatment $k$, i.e., the allocation probability is defined as

$$
p_{m+1, k}=\frac{Y_{m, k}}{\left|\boldsymbol{Y}_{m}\right|}, k=1, \ldots, K,
$$

where $\left|\boldsymbol{Y}_{m}\right|=Y_{m, 1}+\cdots+Y_{m, K}$. We then wait for observing a random variable $\boldsymbol{\xi}(m+1)$, the response of the treatment $k$ at the $(m+1)$-th patient. After that, additional $D_{k, q}(m+1) \geq 0$ balls of type $q, q=1, \ldots, K$, are added to the urn, where $D_{k, q}(m+1)$ is a function of $\boldsymbol{\xi}(m+1)$ and also may be a function of urn compositions, assignments and responses of previous stages. This procedure is repeated through out $n$ stages. This relation can be written as the following recursive formula:

$$
\boldsymbol{Y}_{m}=\boldsymbol{Y}_{m-1}+\boldsymbol{X}_{m} \boldsymbol{D}_{m}
$$

where $\boldsymbol{D}_{m}=\left(D_{k, q}(m)\right)_{k, q=1}^{K}$, and $\boldsymbol{X}_{m}$ is the result of the $m$-th draw, distributed according to the urn composition at the previous stage, i.e., if the $m$ th draw is a type $k$ ball, then the 
$k$ th component of $\boldsymbol{X}_{m}$ is 1 and other components are 0. The matrices $\boldsymbol{D}_{m}$ 's are referred to as the adding rules. The conditional expectations $\boldsymbol{H}_{m}=\left(\mathrm{E}\left[D_{k, q}(m) \mid \mathscr{F}_{m-1}\right]\right)_{k, q=1}^{d}$, given the history sigma field $\mathscr{F}_{m-1}$ generated by the urn compositions $\boldsymbol{Y}_{1}, \ldots, \boldsymbol{Y}_{m-1}$, the assignments $\boldsymbol{X}_{1}, \ldots, \boldsymbol{X}_{m-1}$ and the responses $\boldsymbol{\xi}(1), \ldots, \boldsymbol{\xi}(m-1)$ of all previous stages, $m=1,2, \ldots$, are referred to as the generating matrices. When $\boldsymbol{D}_{m}, m=1,2, \ldots$, are independent and identically distributed, the GFU model is usually said to be homogeneous. In such case $\boldsymbol{H}_{m}=\boldsymbol{H}$ are identical and nonrandom, and usually the adding rule $\boldsymbol{D}_{m}$ is merely a function of the $m$ th patient's observed outcome. In the general heterogeneous cases, both $\boldsymbol{D}_{m}$ and $\boldsymbol{H}_{m}$ depend on the entire history of all previous trials which provides more information of the efficacy of the treatments. However, we should suppose that $\boldsymbol{H}_{m}$ will converge to a matrix $\boldsymbol{H}$.

The randomized play-the-winner rule is a homogeneous urn model with

$$
\boldsymbol{D}_{m}=\left(\begin{array}{cc}
\xi_{m, 1} & 1-\xi_{m, 1} \\
1-\xi_{m, 2} & \xi_{m, 2}
\end{array}\right), \quad \boldsymbol{H}=\left(\begin{array}{cc}
p_{1} & q_{1} \\
q_{2} & p_{2}
\end{array}\right),
$$

where $\xi_{m, k}=1$ if the response of the $m$-th patient on treatment $k$ is a success, and $=0$ for otherwise, $p_{k}$ is the success probability of treatment $k, q_{k}=1-p_{k}, k=1,2$.

For considering the asymptotic properties of $\boldsymbol{N}_{n}$, we suppose that $\boldsymbol{H}$ has a simple largest eigenvalue $\beta>0$ and the corresponding right eigenvector $\boldsymbol{v}=\left(v_{1}, \ldots, v_{K}\right)$ and the left eigenvector $\boldsymbol{u}^{\prime}=\left(u_{1}, \ldots, u_{K}\right)^{\prime}$ with $\sum_{k} v_{k}=\sum_{k} v_{k} u_{k}=1$ and $v_{k}>0, u_{k}>0, k=1, \ldots, K$. Define $\Delta \boldsymbol{M}_{m, 1}=\boldsymbol{X}_{m}-\mathrm{E}\left[\boldsymbol{X}_{m} \mid \mathscr{F}_{m-1}\right], \Delta \boldsymbol{M}_{m, 2}=\boldsymbol{X}_{m}\left(\boldsymbol{D}_{m}-\boldsymbol{H}_{m}\right)$ and $\overline{\boldsymbol{H}}=\boldsymbol{H}-\beta \mathbf{1}^{\prime} \boldsymbol{v}$, where $\mathbf{1}=(1, \ldots, 1)$. It can be shown that $\left|\boldsymbol{Y}_{m}\right| \sim \beta m$ and

$$
\begin{aligned}
\Delta \boldsymbol{Y}_{m}= & \boldsymbol{Y}_{m}-\boldsymbol{Y}_{m-1} \\
= & \boldsymbol{X}_{m}\left(\boldsymbol{D}_{m}-\boldsymbol{H}_{m}\right)+\left(\boldsymbol{X}_{m}-\mathrm{E}\left[\boldsymbol{X}_{m} \mid \mathscr{F}_{m-1}\right]\right) \boldsymbol{H}_{m}+\frac{\boldsymbol{Y}_{m-1}}{\left|\boldsymbol{Y}_{m-1}\right|} \boldsymbol{H}_{m} \\
= & \boldsymbol{v}+\Delta \boldsymbol{M}_{m, 1}\left(\boldsymbol{H}_{m}-\beta \mathbf{1}^{\prime} \boldsymbol{v}\right)+\Delta \boldsymbol{M}_{m, 2}+\left(\frac{\boldsymbol{Y}_{m-1}}{\left|\boldsymbol{Y}_{m-1}\right|}-\boldsymbol{v}\right)\left(\boldsymbol{H}-\beta \mathbf{1}^{\prime} \boldsymbol{v}\right) \\
& +\left(\frac{\boldsymbol{Y}_{m-1}}{\left|\boldsymbol{Y}_{m-1}\right|}-\boldsymbol{v}\right)\left(\boldsymbol{H}_{m}-\boldsymbol{H}\right)+\boldsymbol{v}\left(\boldsymbol{H}_{m}-\boldsymbol{H}\right) \\
\approx & \beta \boldsymbol{v}+\Delta \boldsymbol{M}_{m, 1}\left(\boldsymbol{H}_{m}-\beta \mathbf{1}^{\prime} \boldsymbol{v}\right)+\Delta \boldsymbol{M}_{m, 2}+\left(\frac{\boldsymbol{Y}_{m-1}}{\beta(m-1)}-\boldsymbol{v}\right) \overline{\boldsymbol{H}}+\boldsymbol{v}\left(\boldsymbol{H}_{m}-\boldsymbol{H}\right)
\end{aligned}
$$

If we ignore the remainder $\boldsymbol{v}\left(\boldsymbol{H}_{m}-\boldsymbol{H}\right)$ and replace the martingales $\boldsymbol{M}_{n, 1}$ and $\boldsymbol{M}_{n, 2}$ by two (independent) Brownian motions $\boldsymbol{B}_{1}(n) \boldsymbol{\Sigma}_{1}^{1 / 2}$ and $\boldsymbol{B}_{2}(n) \boldsymbol{\Sigma}_{2}^{1 / 2}$, we conclude that

$$
\boldsymbol{Y}_{n}-\beta n \boldsymbol{v} \approx \boldsymbol{G}_{1}(n) \overline{\boldsymbol{H}}+\boldsymbol{G}_{2}(n)
$$


with $\boldsymbol{G}_{j}(t)$ satisfying the stochastic differential equation (SDE):

$$
d \boldsymbol{G}_{j}(t)=d \boldsymbol{B}_{j}(t) \boldsymbol{\Sigma}_{j}^{1 / 2}+\frac{\boldsymbol{G}_{j}(t)}{t} \frac{\overline{\boldsymbol{H}}}{\beta} d t, \quad \boldsymbol{G}_{j}(0)=\mathbf{0} \text { or } \boldsymbol{G}_{j}(1)=\mathbf{0}
$$

where $\boldsymbol{B}_{1}$ and $\boldsymbol{B}_{2}$ are two independent standard Brownian motions, $\boldsymbol{\Sigma}_{1}=\operatorname{diag}(\boldsymbol{v})-\boldsymbol{v}^{\prime} \boldsymbol{v}$ and $\boldsymbol{\Sigma}_{2}=\lim _{m \rightarrow \infty}\left(\sum_{k=1}^{K} v_{k} \operatorname{Var}\left\{\boldsymbol{D}_{m}^{(k)} \mid \mathscr{F}_{m-1}\right\}\right)$ and $\boldsymbol{D}_{m}^{(k)}$ is the $k$ row of $\boldsymbol{D}_{m}$. Further,

$$
\begin{aligned}
& \Delta\left(\boldsymbol{N}_{t}-t \boldsymbol{v}\right)=\Delta \boldsymbol{M}_{t, 1}+\frac{\boldsymbol{Y}_{t-1}}{\left|\boldsymbol{Y}_{t-1}\right|}-\boldsymbol{v} \\
\approx & d \boldsymbol{B}_{1}(t) \boldsymbol{\Sigma}_{1}^{1 / 2}+\frac{\boldsymbol{G}_{1}(t) \overline{\boldsymbol{H}}+\boldsymbol{G}_{2}(t)}{\beta t} d t=d \boldsymbol{G}_{1}(t)+\frac{\boldsymbol{G}_{2}(t)}{\beta t} d t,
\end{aligned}
$$

So,

$$
\boldsymbol{N}_{n}-n \boldsymbol{v} \approx \boldsymbol{G}_{1}(n)+\int_{*}^{n} \frac{\boldsymbol{G}_{2}(t)}{\beta t} d t
$$

By calculating the variance of the Gaussian processes $\boldsymbol{G}_{1}(t)$ and $\boldsymbol{G}_{2}(t)$, one can derive the asymptotic normality of $\boldsymbol{Y}_{n}$ and $\boldsymbol{N}_{n}$.

For details on the asymptotic normalities of urn models, one can refer to Bai, $\mathrm{Hu}$ and Rosneberger (2002), Hu and Zhang (2004b), Bai and $\mathrm{Hu}$ (2005), Zhang and $\mathrm{Hu}$ (2009), Zhang (2012) etc. Among these studies, Bai, Hu and Zhang (2002), Hu and Zhang (2004b), Zhang and $\mathrm{Hu}$ (2009), Zhang (2012) studied the asymptotic properties via the Gaussian approximation. The following Theorem 3.1 and Corollary 3.1 give a summary of theses results.

Theorem 3.1 Suppose $\sup _{m} E\left\|\boldsymbol{D}_{m}\right\|^{2+\delta}<\infty$. Let $\lambda_{1}=\beta, \lambda_{2}, \ldots, \lambda_{K}$ be the eigenvalues of $\boldsymbol{H}$, and $\lambda=\max \left\{\operatorname{Re}\left(\lambda_{2}\right) / \lambda_{1}, \ldots, \operatorname{Re}\left(\lambda_{K}\right) / \lambda_{1}\right\}$.

(i) If $\lambda<1$ and $\sum_{m=1}^{n}\left\|\boldsymbol{H}_{m}-\boldsymbol{H}\right\|=o(n)$ a.s., then

$$
\frac{N_{n, k}}{n} \rightarrow \beta v_{k} \text { a.s. and } \frac{Y_{n, k}}{Y_{n, 1}+\cdots Y_{n, K}} \rightarrow v_{k} \text { a.s. }
$$

(ii) If $\lambda<1 / 2$ and

$$
\sum_{m=1}^{n}\left\|\boldsymbol{H}_{m}-\boldsymbol{H}\right\|=o(\sqrt{n}) \text { a.s. }
$$

then

$$
\sqrt{n}\left(\frac{\boldsymbol{Y}_{n}}{n}-\beta \boldsymbol{v}\right) \stackrel{\mathcal{D}}{\rightarrow} N\left(\mathbf{0}, \boldsymbol{\Gamma}_{1}\right) \text { and } \sqrt{n}\left(\frac{\boldsymbol{N}_{n}}{n}-\boldsymbol{v}\right) \stackrel{\mathcal{D}}{\rightarrow} N\left(\mathbf{0}, \boldsymbol{\Gamma}_{2}\right)
$$

(iii) If $\lambda=1 / 2$ and $\sum_{m=1}^{n}\left\|\boldsymbol{H}_{m}-\boldsymbol{H}\right\| / \sqrt{m}=o(\sqrt{\log n})$ a.s., then

$$
\frac{\sqrt{n}}{(\log n)^{\nu-1 / 2}}\left(\frac{\boldsymbol{Y}_{n}}{n}-\beta \boldsymbol{v}\right) \stackrel{\mathcal{D}}{\rightarrow} N\left(\mathbf{0}, \boldsymbol{\Gamma}_{1}^{*}\right) \text { and } \frac{\sqrt{n}}{(\log n)^{\nu-1 / 2}}\left(\frac{\boldsymbol{N}_{n}}{n}-\boldsymbol{v}\right) \stackrel{\mathcal{D}}{\rightarrow} N\left(\mathbf{0}, \boldsymbol{\Gamma}_{2}^{*}\right) \text {. }
$$


(iv) If $1 / 2<\lambda<1$ and $\sum_{m=1}^{n}\left\|\boldsymbol{H}_{m}-\boldsymbol{H}\right\|=o\left(n^{\lambda-\epsilon}\right)$ a.s., then

$$
\left\|\boldsymbol{Y}_{n}-\beta n \boldsymbol{v}\right\|=O\left(n^{\lambda} \log ^{\nu-1} n\right) \text { a.s. and }\left\|\boldsymbol{N}_{n}-n \boldsymbol{v}\right\|=O\left(n^{\lambda} \log ^{\nu-1} n\right) \text { a.s. }
$$

Here $\nu$ is the largest order of the Jordan blocks with respect to the eigenvalues $\lambda_{s}$ with $\operatorname{Re}\left(\lambda_{s}\right) / \lambda_{1}=\lambda$.

For the randomized play-the-winner rule, we have $\beta=1, \lambda=p_{1}+p_{2}-1$. The problem is reduced to the one-dimensional problem: $Y_{n, 1}-n v_{1} \approx G_{1}(n) \lambda+G_{2}(n), N_{n, 1}-n v_{1} \approx$ $G_{1}(n)+\int_{*}^{n} \frac{G_{2}(t)}{t} d t$

$$
d G_{j}(t)=\sigma_{j} d B_{j}(t)+\lambda \frac{G_{j}(t)}{t} d t
$$

with $\sigma_{1}^{2}=v_{1} v_{2}=\frac{q_{1} q_{2}}{\left(q_{1}+q_{2}\right)^{2}}$ and $\sigma_{2}^{2}=v_{1} p_{1} q_{1}+v_{2} p_{2} q_{2}=\frac{q_{1} q_{2}\left(p_{1}+p_{2}\right)}{q_{1}+q_{2}}$.

Corollary 3.1 (a) If $p_{1}+p_{2}<1.5$, then $\sqrt{n}\left(N_{n, 1} / n-q_{2} /\left(q_{1}+q_{2}\right)\right) \stackrel{\mathcal{D}}{\rightarrow} N\left(0, \sigma_{R P W}^{2}\right)$, where

$$
\begin{gathered}
\sigma_{R P W}^{2}=\frac{q_{1} q_{2}\left[5-2\left(q_{1}+q_{2}\right)\right]}{\left[2\left(q_{1}+q_{2}\right)-1\right]\left(q_{1}+q_{2}\right)^{2}}, \quad \text { and } \\
\sqrt{n}\left(\frac{Y_{n, 1}}{Y_{n, 1}+Y_{n, 2}}-\frac{q_{2}}{q_{1}+q_{2}}\right) \stackrel{\mathcal{D}}{\rightarrow} N\left(0, \frac{q_{1} q_{2}}{\left(2\left(q_{1}+q_{2}\right)-1\right)\left(q_{1}+q_{2}\right)^{2}}\right) .
\end{gathered}
$$

(b) If $p_{1}+p_{2}=1.5$, then

$$
\begin{gathered}
\sqrt{n / \log n}\left(Y_{n, 1} / n-q_{2} /\left(q_{1}+q_{2}\right)\right) \stackrel{\mathcal{D}}{\rightarrow} N\left(0,4 q_{1} q_{2}\right), \quad \text { and } \\
\sqrt{n / \log n}\left(N_{n, 1} / n-q_{2} /\left(q_{1}+q_{2}\right)\right) \stackrel{\mathcal{D}}{\rightarrow} N\left(0,16 q_{1} q_{2}\right) .
\end{gathered}
$$

(c) If $p_{1}+p_{2}>1.5$, then there exists a random variable $\xi$ such that

$$
\begin{gathered}
n^{p_{1}+p_{2}-1}\left(Y_{n, 1}-n q_{2} /\left(q_{1}+q_{2}\right)\right) \rightarrow \xi / 2 \text { a.s., and } \\
n^{p_{1}+p_{2}-1}\left(N_{n, 1}-n q_{2} /\left(q_{1}+q_{2}\right)\right) \rightarrow \xi \text { a.s. }
\end{gathered}
$$

As a multi-treatment extension of the RPW rule, Wei (1979) proposed a GPU model to allocate patients, in which the urn is updated in the following way: at the $m$ th stage, if a patient is assigned to treatment $k$ and the outcome is a success, then a type $k$ ball is added to the urn, otherwise, if the treatment $k$ for a patient is a failure, then $1 /(K-1)$ balls are added to the urn for each of other $(K-1)$ types. This urn model is a homogenous 
urn model with $\boldsymbol{H}=\left\{h_{k, j}\right\}$, where $h_{k, k}=p_{k}$ and $h_{k, j}=q_{k} /(K-1)(j \neq k)$, and $q_{k}$ is the failure rate of treatment $k, p_{k}=1-p_{k}$ is the success probability. So, $v_{k}=\frac{1 / q_{k}}{\sum_{j=1}^{K}\left(1 / q_{j}\right)}$.

One important class of the non-homogenous urn model is the sequential estimationadjusted urn model proposed by Zhang, Hu and Cheung (2006), in which the urn is updated according to the current response and the current estimate $\widehat{\boldsymbol{\theta}}_{m}$ of the parameter $\boldsymbol{\theta}$, and so $\boldsymbol{H}\left(\widehat{\boldsymbol{\theta}}_{m}\right)$ is a function of the current estimator. In this case, the fastest convergence rate of $\boldsymbol{H}_{m}-\boldsymbol{H}$ is $O_{P}(\sqrt{m})$ so that the condition (3.6) is not satisfied and the term $\boldsymbol{v}\left(\boldsymbol{H}_{m}-\boldsymbol{H}\right)$ can not be ignored. In this case,

$$
v\left(\boldsymbol{H}_{m}-\boldsymbol{H}\right) \approx\left(\widehat{\boldsymbol{\theta}}_{m}-\boldsymbol{\theta}\right) \frac{\partial[\boldsymbol{v} \boldsymbol{H}(\boldsymbol{\theta})]}{\partial \boldsymbol{\theta}} \approx \frac{\boldsymbol{W}_{3}(m)}{m} \frac{\partial[\boldsymbol{v} \boldsymbol{H}(\boldsymbol{\theta})]}{\partial \boldsymbol{\theta}}
$$

where $\boldsymbol{W}_{3}(t)$ is another Brownian motion which is independent of $\boldsymbol{B}_{1}(\cdot)$, but may depend on $\boldsymbol{B}_{2}(\cdot)$. The variance-covariance of $\left(\boldsymbol{B}_{1}(t) \boldsymbol{\Sigma}_{1}^{1 / 2}, \boldsymbol{B}_{2}(t) \boldsymbol{\Sigma}_{2}^{1 / 2}, \boldsymbol{W}_{3}(t)\right)$ is given in (5.23) of Zhang, Hu and Cheung (2006).

Hereafter, for a vector function $\boldsymbol{g}(\boldsymbol{x})$, its derivative $\frac{\partial g(\boldsymbol{x})}{\partial \boldsymbol{x}}=\left(\frac{\partial g_{k}(\boldsymbol{x})}{\partial x_{j}} ; j, k=1, \ldots, K\right)$ is a matrix. In the approximation (3.3) and (3.5), $\boldsymbol{G}_{2}(t)$ should be replaced with $\boldsymbol{G}_{2}(t)+\boldsymbol{G}_{3}(t)$ and

$$
d \boldsymbol{G}_{3}(t)=\frac{\boldsymbol{W}_{3}(t)}{t} d t+\frac{\boldsymbol{G}_{3}(t)}{t} \frac{\overline{\boldsymbol{H}}}{\beta} d t .
$$

The conclusions of Theorem 3.1 remains true with a different group of variance-covariance matrices.

In particular, suppose $\widehat{\boldsymbol{\theta}}_{m}=\left(\widehat{\boldsymbol{\theta}}_{m, 1}, \ldots, \widehat{\boldsymbol{\theta}}_{m, K}\right)$ is the maximum likelihood estimator of the parameter $\boldsymbol{\theta}=\left(\boldsymbol{\theta}_{1}, \ldots, \boldsymbol{\theta}_{K}\right)$ based on the responses available up to stage $m$. If we define

$$
\boldsymbol{D}_{m}=\boldsymbol{H}_{m}=\beta \mathbf{1}^{\prime} \boldsymbol{\rho}\left(\widehat{\boldsymbol{\theta}}_{m}\right) \text { with } \rho_{k}(\cdot)>0 \text { and } \sum_{k} \rho_{k}(\cdot)=1, \beta>0
$$

then $\boldsymbol{N}_{n}-n \boldsymbol{v}=O(\sqrt{n \log \log n})$ a.s. and

$$
\sqrt{n}\left(\frac{\boldsymbol{N}_{n}}{n}-\boldsymbol{\rho}\right) \stackrel{\mathcal{D}}{\rightarrow} N\left(\mathbf{0}, \operatorname{diag}(\boldsymbol{\rho})-\boldsymbol{\rho}^{\prime} \boldsymbol{\rho}+6 \boldsymbol{\Sigma}_{L B}\right),
$$

where $\boldsymbol{\rho}=\boldsymbol{\rho}(\boldsymbol{\theta})$,

$$
\boldsymbol{\Sigma}_{L B}=\left(\frac{\partial \boldsymbol{\rho}(\boldsymbol{\theta})}{\partial \boldsymbol{\theta}}\right)^{\prime} d a i g\left(\frac{\boldsymbol{I}_{1}^{-1}\left(\boldsymbol{\theta}_{1}\right)}{\rho_{1}(\boldsymbol{\theta})}, \cdots, \frac{\boldsymbol{I}_{K}^{-1}\left(\boldsymbol{\theta}_{K}\right)}{\rho_{K}(\boldsymbol{\theta})}\right) \frac{\partial \boldsymbol{\rho}(\boldsymbol{\theta})}{\partial \boldsymbol{\theta}},
$$

and $\boldsymbol{I}_{k}\left(\boldsymbol{\theta}_{k}\right)$ is the Fisher information for a single observation on treatment $k=1, \ldots, K$. Hereafter, $\boldsymbol{I}_{k}^{-1}$ denotes the inverse matrix of $\boldsymbol{I}_{k}$. 
When the estimator $\widehat{\boldsymbol{\theta}}_{m}$ of the parameter $\boldsymbol{\theta}$ is utilized to update the allocation probabilities, the adaptive randomization scheme can start only after an initial estimator is defined because at the first few steps there are insufficient data for estimating $\boldsymbol{\theta}$. In general, there are three ways to overcome this problem: 1$)$ initial $K m_{0}$ patients $\left(m_{0}\right.$ is some small positive integer) are randomized to treatments $1, \ldots, K$ by means of some restricted randomization design, and their outcomes are used to estimate $\boldsymbol{\theta} ; 2$ ) choose an initial value $\boldsymbol{\theta}_{0}$ as the estimator until sufficient amount of data are collected to estimate $\boldsymbol{\theta}$ (the value $\boldsymbol{\theta}_{0}$ is usually a guess value of the parameter or an estimate from other trials); 3) apply the Bayesian estimation method.

\subsection{Drop-the-Loser}

The asymptotic normality for the randomized play-the-winner rule as well as its extensions to generalized Pólya urn models can be obtained only when the condition $\rho \leq 1 / 2$ is satisfied, and the variabilities are very large. Many of these designs are slow to converge and produce less powerful treatment comparison hypothesis tests. In the family of urn models, a major advance was made by Ivanova (2003), who introduced "the drop-the-loser rule", an urn model design with the same limiting allocation as the RPW rule but with much lower variability. In the drop-the-loser rule, an urn is considered with balls of $(K+1)$ types, type $0,1, \ldots, K$, when comparing $K$ treatments. Types $1, \ldots, K$ are called treatment types, and type 0 is called the immigration type. When a patient is ready for randomization, a ball is drawn at random. If it is of a treatment type, the corresponding treatment is assigned and the patient's response is observed. If the response is a success, the ball is replaced and the urn remained unchanged. If a failure the ball is not replaced. When an immigration type ball is drawn, no treatment assignment is made, and the ball is return to the urn together with one ball of each treatment type. Ivanova $(2003,2006)$ showed the asymptotic normality by embedding the urn process to a death-and-immigration process.

Theorem 3.2 Let $\boldsymbol{v}=\left(v_{1}, \cdots, v_{K}\right)$ with $v_{k}=\frac{1 / q_{k}}{\sum_{j=1}^{K}\left(1 / q_{j}\right)}$. Then

$$
\sqrt{n}\left(\frac{\boldsymbol{N}_{n}}{n}-\boldsymbol{v}\right) \stackrel{D}{\rightarrow} N\left(\mathbf{0}, \boldsymbol{\Sigma}_{D L}\right)
$$

with $\boldsymbol{\Sigma}_{D L}=\left(\boldsymbol{I}-\boldsymbol{v}^{\prime} \mathbf{1}\right) \operatorname{diag}\left(\frac{v_{1} p_{1}}{q_{1}}, \cdots, \frac{v_{K} p_{K}}{q_{K}}\right)\left(\boldsymbol{I}-\mathbf{1}^{\prime} \boldsymbol{v}\right)$. It can be verified that

$$
\boldsymbol{\Sigma}_{D L}=\left(\frac{\partial \boldsymbol{v}}{\partial \boldsymbol{q}}\right)^{\prime} \operatorname{diag}\left(\frac{p_{1} q_{1}}{v_{1}}, \cdots, \frac{p_{K} q_{K}}{v_{K}}\right) \frac{\partial \boldsymbol{v}}{\partial \boldsymbol{q}} .
$$


In particular, for the two-treatment case,

$$
\sqrt{n}\left(\frac{N_{n, 1}}{n}-\frac{q_{2}}{q_{1}+q_{2}}\right) \stackrel{D}{\rightarrow} N\left(0, \frac{q_{1} q_{2}\left(p_{1}+p_{2}\right)}{\left(q_{1}+q_{2}\right)^{3}}\right) .
$$

The asymptotic normality of DL rule holds for all cases of $0<p_{1}, p_{2}<1$ and the variability $\frac{q_{1} q_{2}\left(p_{1}+p_{2}\right)}{\left(q_{1}+q_{2}\right)^{3}}$ is much smaller than the one of the RPW rule. For the multi-treatment case, the formula of $\boldsymbol{\Sigma}_{L D}$ in Ivanova (2006) is given in a different expression. The present one is due to Zhang et al. (2011).

\subsection{Generalized Drop-the-Loser and Immigrated Urn Model}

Generalizations of the drop-the-loser rule can be found in Zhang et al. (2007), Sun, Cheung and Zhang (2007) and Zhang et al. (2011). Zhang et al. (2011) proposed an immigrated urn model which provides a unified theory of urn models for clinical trials. In an immigrated urn model, as in the drop-the-loser rule, the urn contains balls of $(K+1)$ types, where types $1, \ldots, K$ stand for treatment types, and type 0 stands for the immigration type. After $m$ assignments, suppose the urn composition is $\left(Y_{m, 0}, Y_{m, 1}, \ldots, Y_{m, K}\right)$. For the $(m+1)$-th patient's treatment assignment, a ball is drawn at random. If an immigration type ball is drawn, no assignment is made, and the ball is return to the urn together with $a_{m+1, k} \geq 0$ balls of type $k, k=1, \ldots, K$. The process is repeated until a ball of a treatment type is drawn. If the ball drawn is of type $k(k=1, \ldots, K)$, the corresponding treatment is assigned and the patient's response $\boldsymbol{\xi}(m+1)$ is observed. The ball is also return with additional $D_{k, j}(m+1)$ balls of each treatment type $j, j=1, \ldots, K . D_{k, j}(m+1)$ is a function of the response $\boldsymbol{\xi}(m+1)=\left(\xi_{1}(m+1), \ldots, \xi_{K}(m+1)\right)$. In the IMU model, the diagonal elements of $\boldsymbol{D}_{m+1}$ allow negative values, which means the dropping of balls, and

$a_{m+1, k}=a_{k}\left(\widehat{\boldsymbol{\theta}}_{m}\right)$ can be a function of the current estimator $\widehat{\boldsymbol{\theta}}_{m}$ of the parameter $\boldsymbol{\theta}$. The vector $\boldsymbol{a}_{m}=\left(a_{m, 1}, \ldots, a_{m, K}\right)$ are called the immigration rates. When $a_{m, k} \equiv 0$ and $Y_{0,0}=0$, the IMU reduces to GPU. When $\boldsymbol{D}_{m}$ is diagonal and its elements have negative means, the IMU is the generalized drop-the-loser rule proposed by Zhang et al. (2007) and Sun, Cheung and Zhang (2007). Here when the urn allows balls with negative numbers, we assume that the balls of a type with a negative number have no-chance to be selected and so the selection probabilities are the proportions of positive numbers of balls in the urn.

For considering the theory of the IMU model, without loss of generality, we suppose the 
parameter $\theta_{k}$ is one-dimensional and the mean of the response $\xi_{k}(m)$ and the estimator $\widehat{\theta}_{m, k}$ is the current sample mean, and assume that $\left\{\left(\boldsymbol{D}_{m}, \boldsymbol{\xi}(m)\right)\right\}$ are i.i.d. Let $\boldsymbol{H}=\mathrm{E}\left[\boldsymbol{D}_{m}\right]$ be the mean matrix as in the GPU model. If $\boldsymbol{H} \mathbf{1}^{\prime}=\beta \mathbf{1}^{\prime}$ with $\beta>0$, i.e., at each stage the average of the total number of balls added according the treatments are positive, then the total number of balls in the urn gradually increases to infinity. Hence, the probability of drawing an immigration ball drops to zero. For this case, the IMU model is asymptotically equivalent to the generalized Pólya urn model without immigration, and the conclusions of Theorem 3.1 remain true. When $\boldsymbol{H} \mathbf{1}^{\prime}<\mathbf{0}^{\prime}$, the urn composition is mainly updated by the immigration, and we have the following theorem.

Theorem 3.3 Let $\boldsymbol{A}=(-\boldsymbol{H})^{-1}\left(\boldsymbol{I}-\mathbf{1}^{\prime} \boldsymbol{v}\right)$,

$$
\begin{gathered}
\boldsymbol{v}(\boldsymbol{\theta})=\left(v_{1}, \ldots, v_{K}\right)=\frac{\boldsymbol{a}(\boldsymbol{\theta})(-\boldsymbol{H})^{-1}}{\boldsymbol{a}(\boldsymbol{\theta})(-\boldsymbol{H})^{-1} \mathbf{1}^{\prime}}, \\
\boldsymbol{\Sigma}_{11}=\sum_{k=1}^{K} v_{k} \operatorname{Var}\left\{\boldsymbol{D}_{1}^{(k)}\right\}, \quad \boldsymbol{\Sigma}_{D}=\boldsymbol{A}^{\prime} \boldsymbol{\Sigma}_{11} \boldsymbol{A}, \\
\boldsymbol{\Sigma}_{12}=\left(\operatorname{Cov}\left\{D_{1, k j}, \xi_{k}\right\} ; j, k=1, \ldots, K\right), \quad \boldsymbol{\Sigma}_{D \xi}=\boldsymbol{A}^{\prime} \boldsymbol{\Sigma}_{12} \frac{\partial \boldsymbol{v}(\boldsymbol{\theta})}{\partial \boldsymbol{\theta}}, \\
\boldsymbol{\Sigma}_{\xi}=\left(\frac{\partial \boldsymbol{v}(\boldsymbol{\theta})}{\partial \boldsymbol{\theta}}\right)^{\prime} \operatorname{diag}\left(\frac{\operatorname{Var}\left\{\xi_{1,1}\right\}}{v_{1}}, \ldots, \frac{\operatorname{Var}\left\{\xi_{1, K}\right\}}{v_{K}}\right) \frac{\partial \boldsymbol{v}(\boldsymbol{\theta})}{\partial \boldsymbol{\theta}}
\end{gathered}
$$

and $\boldsymbol{\Sigma}=\boldsymbol{\Sigma}_{D}+2 \boldsymbol{\Sigma}_{\xi}+\boldsymbol{\Sigma}_{D \xi}+\boldsymbol{\Sigma}_{D \xi}^{\prime}$. Suppose $E\left\|\boldsymbol{D}_{m}\right\|^{2+\epsilon}<\infty, E\|\boldsymbol{\xi}(m)\|^{2+\epsilon}<\infty$, and $\boldsymbol{H} \mathbf{1}^{\prime}<\mathbf{0}^{\prime}, Y_{0,0}>0$. Then

$$
\begin{gathered}
Y_{n, k}=o\left(n^{1 / 2-\epsilon}\right) \text { a.s., } \quad k=1, \ldots, K, \quad \boldsymbol{N}_{n}-n \boldsymbol{v}=O(\sqrt{n \log \log n}) \text { a.s. } \\
\text { and } \sqrt{n}\left(\frac{\boldsymbol{N}_{n}}{n}-\boldsymbol{v}(\boldsymbol{\theta})\right) \stackrel{D}{\rightarrow} N(\mathbf{0}, \boldsymbol{\Sigma}) .
\end{gathered}
$$

In particular,

(i) when $\boldsymbol{D}_{m} \equiv$ const (for example $\boldsymbol{D}_{m}=\operatorname{diag}(-1, \ldots,-1)$ ), one has

$$
\sqrt{n}\left(\frac{\boldsymbol{N}_{n}}{n}-\boldsymbol{v}(\boldsymbol{\theta})\right) \stackrel{D}{\rightarrow} N\left(\mathbf{0}, 2 \boldsymbol{\Sigma}_{\xi}\right)
$$

(ii) when $\boldsymbol{a}(\boldsymbol{\theta}) \equiv$ const and each $D_{m, k j}$ is a linear function of $\xi_{m, k}, j=1, \ldots, K$, so that $\boldsymbol{v}$ is a function of $\theta_{k}=E \xi_{m, k}, k=1, \ldots, k$, one has

$$
\sqrt{n}\left(\frac{\boldsymbol{N}_{n}}{n}-\boldsymbol{v}(\boldsymbol{\theta})\right) \stackrel{D}{\rightarrow} N\left(\mathbf{0}, \boldsymbol{\Sigma}_{\xi}\right)
$$

When $\boldsymbol{H} \mathbf{1}^{\prime}=\mathbf{0}$, we have $\boldsymbol{N}_{n}-n \boldsymbol{v}=O(\sqrt{n \log \log n})$ a.s. and $\boldsymbol{N}_{n}-n \boldsymbol{v}=O(\sqrt{n})$ in probability. The asymptotic normality is still an open problem. 


\subsection{Randomly Reinforced Urn}

Another type of urn models, called randomly reinforced urn (RRU), are also proposed for randomizing patients to treatments. In a response-adaptive design driven by a RRU model, an observation of an outcome from treatment $k$ results in only adding balls of the same type $k$. A RRU procedure leads to an extreme limiting allocation so that the sample allocation proportions of the best treatment converges to 1 , and others converge to zero. For the properties of the RRU design, one may refer to Li, Durham and Flournoy (1996), May and Flournoy (2009), Zhang et al. (2014a) etc. However, the RRU design has very high variability and so is not strongly recommended in the term of power.

\section{Optimal and Efficient RAR Designs}

The RPW rule and many of its extensions based on urn models were proposed by the intuitive motivation of placing more patients on the superior treatment, and they were not designed to optimize any criterion. Hu and Rosenberger (2003) formalized the development of optimal response-adaptive randomization procedures using the following three steps:

1) An optimal allocation is derived as a solution to some formal optimization problem.

2) A response-adaptive randomization procedure is chosen to converge to the optimal target. The procedure should be fully randomized, have minimal variability, and high speed of convergence to the chosen optimal allocation.

3) Operating characteristics of the chosen response-adaptive randomization design are studied theoretically and by simulation under a variety of standard to worst-case scenarios.

\subsection{Optimization}

For two-treatment trials, a general optimization problem is described in Jennison and Turnbull (2000), which led to the development of optimal response-adaptive randomization designs for trials with binary responses (Rosenberger et al. (2001); Ivanova and Rosenberger (2001); Rosenberger and Hu (2004); Baldi Antognini and Giovagnoli (2010)), normal outcomes (Biswas and Mandal (2004); Zhang and Rosenberger (2006); Gwise, Hu and $\mathrm{Hu}$ (2008); Biswas and Bhattacharya (2009, 2010, 2011)), and survival outcomes (Zhang and 
Rosenberger (2007)). The most interesting optimal allocation targets are the Neyman allocation for continuous response and the RSIHR allocation for binary responses.

In Jennison and Turnbull (2000)'s approach, let $\xi_{m, 1}$ arise from a $N\left(\mu_{1}, \sigma_{1}^{2}\right)$ distribution and $\xi_{m, 2}$ arise from a $N\left(\mu_{2}, \sigma_{2}^{2}\right)$ distribution, $m=1,2, \ldots$ For testing the treatment effect $\theta=: \mu_{1}-\mu_{2}=0$, a natural test is the Wald test given by

$$
Z=\frac{\widehat{\mu}_{1}-\widehat{\mu}_{2}}{\sqrt{\frac{\widehat{\sigma}_{1}^{2}}{n_{1}}+\frac{\widehat{\sigma}_{2}^{2}}{n_{2}}}},
$$

where $n_{1}$ and $n_{2}$ are the sample size for treatment 1 and 2 . The (asymptotic) power of the test is a decreasing of

$$
\eta(\theta)=\frac{\sigma_{1}^{2}}{n_{1}}+\frac{\sigma_{2}^{2}}{n_{2}}
$$

Fixing $\eta$ to a constant, say $\delta$, we wish to find the value of $\frac{n_{1}}{n_{1}+n_{2}}$ that minimizes

$$
u(\theta) n_{1}+v(\theta) n_{2},
$$

where $u(\cdot)$ and $v(\cdot)$ are appropriately chosen functions of $\theta$. Because we wish to put more patients on treatment 1 if $\theta>0$ and more patients on treatment 2 if $\theta<0$, Jennison and Turnbull explore functions where $u(\cdot)$ and $v(\cdot)$ are strictly positive, and $u(\theta)$ is decreasing in $\theta$ for $\theta<0$ and $v(\theta)$ is increasing in $\theta$ for $\theta>0$. By using the Lagrange multiplier method and minimizing

$$
u(\theta) n_{1}+v(\theta) n_{2}+\lambda\left(\frac{\sigma_{1}^{2}}{n_{1}}+\frac{\sigma_{2}^{2}}{n_{2}}-K\right),
$$

a minimum is achieved at

$$
\frac{n_{1}}{n_{1}+n_{2}}=\rho_{1}=: \frac{\sigma_{1} / \sqrt{u(\theta)}}{\sigma_{1} / \sqrt{u(\theta)}+\sigma_{2} / \sqrt{v(\theta)}} .
$$

If $u(\cdot) \equiv v(\cdot) \equiv 1$, then we have $\rho_{1}=\sigma_{1} /\left(\sigma_{1}+\sigma_{2}\right)$, which is the Neyman allocation. This allocation maximizes the power of the usual $Z$-test for fixed sample size $n_{1}+n_{2}=n$.

In the case of the binary responses, for testing the equality of treatment effects, the Wald test is given by

$$
Z=\frac{\widehat{p}_{1}-\widehat{p}_{2}}{\sqrt{\frac{\widehat{p}_{1} \widehat{q}_{1}}{n_{1}}+\frac{\widehat{p}_{2} \widehat{q_{2}}}{n_{2}}}},
$$

where $\widehat{p}_{k}$ is the estimator of the cured rate $p_{k}$, and $\widehat{q}_{k}=1-\widehat{p}_{k}, k=1,2$. Rosenberger et al. (2001) suggested fixing the asymptotic variance $\eta\left(q_{1}, q_{2}\right)=\frac{p_{1} q_{1}}{n_{1}}+\frac{p_{2} q_{2}}{n_{2}}$ to a constant, say $\delta$, and minimizing the average failure number $q_{1} n_{1}+q_{2} n_{2}$. The minimum is achieved at

$$
\frac{n_{1}}{n_{1}+n_{2}}=\rho_{1}=: \frac{\sqrt{p_{1}}}{\sqrt{p_{1}}+\sqrt{p_{2}}}
$$


We refer to this as RSIHR allocation.

Many other optimal allocations can be found in literature. For example, Zhang and Rosenberger (2006) proposed the following allocation for normal responses with positive means by minimizing the mean total response for fixed power:

$$
\rho_{1}(\boldsymbol{\theta})=\frac{\sqrt{\mu_{2}} \sigma_{1}}{\sqrt{\mu_{2}} \sigma_{1}+\sqrt{\mu_{1}} \sigma_{2}}
$$

By minimizing the total number of patients with normal response greater than a constant given $c$, Biswas and Mandal (2004) obtained an allocation as

$$
\rho_{1}(\boldsymbol{\theta})=\frac{\sqrt{\Phi\left(\frac{u_{2}-c}{\sigma_{2}}\right)} \sigma_{1}}{\sqrt{\Phi\left(\frac{u_{2}-c}{\sigma_{2}}\right)} \sigma_{1}+\sqrt{\Phi\left(\frac{u_{1}-c}{\sigma_{1}}\right)} \sigma_{1}} .
$$

For multi-arm clinical trials, Tymofyeyev, Rosenberger and $\mathrm{Hu}$ (2007) introduced a general approach for finding allocations to minimize a weighted sum of treatment sample sizes subject to minimal constraints on the power of a homogeneity test and treatment proportions in the trial. Let $\phi\left(n_{1}, \ldots, n_{K}\right)$ be the noncentrality parameter of a suitable multivariate test statistics of interest under the alterative hypothesis, where $n_{k}$ is sample size for treatment $k, n_{1}+\cdots n_{K}=n$. We assume that the noncerntrality parameter is a concave function with nonnegative gradient. Tymofyeyev, Rosenberger and $\mathrm{Hu}$ (2007) suggested considering a general optimization problem:

$$
\begin{aligned}
\min _{n_{1}, \ldots, n_{K}} & \sum_{j=1}^{K} w_{j} n_{j}, \\
\text { such that } & \frac{n_{k}}{\sum_{j=1}^{K} n_{j}} \geq B, \quad k=1, \ldots, K, \\
& \phi\left(n_{1}, \ldots, n_{K}\right) \geq C,
\end{aligned}
$$

or equivenlently,

$$
\begin{aligned}
\max _{m_{1}, \ldots, m_{K}} & \phi\left(m_{1}, \ldots, m_{K}\right) \\
\text { such that } & \frac{m_{k}}{\sum_{j=1}^{K} m_{j}} \geq B, \quad k=1, \ldots, K, \\
& \sum_{j=1}^{K} w_{j} m_{j} \leq M,
\end{aligned}
$$

to derive the allocation target $\rho_{k}=n_{k} / \sum_{j=1}^{K} n_{j}=m_{k} / \sum_{j=1}^{K} m_{j}$. Here $w_{j}$ are positive weights, and the nonnegative constant $B$ with $K B \leq 1$ is a lower bound for the proportion 
$n_{k} / \sum_{j=1}^{K} n_{j}$ (resp. $m_{k} / \sum_{j=1}^{K} m_{j}$ ) that allows us to control explicitly the feasible region of the problem. Under this framework, Jeon and $\mathrm{Hu}$ (2010), for binary trials with $K=3$ treatments, obtained the analytic solution for an allocation minimizing expected treatment failures in the trial subject to minimal constraints on power and treatment proportions. Similar results were obtained by Zhu and $\mathrm{Hu}$ (2009) for exponential outcomes and by Sverdlov, Tymofyeyev and Wong (2011) for censored exponential outcomes.

Also, there is a lot papers considering the $D$-optimization, $D A$-optimization etc. In general, let $\boldsymbol{M}(\boldsymbol{\rho}, \boldsymbol{\theta})$ denote the Fisher information matrix for $\boldsymbol{\theta}$ given a design allocation $\boldsymbol{\rho}=\left(\rho_{1}, \ldots, \rho_{K}\right)$. By minimizing $\boldsymbol{M}^{-1}(\boldsymbol{\rho}, \boldsymbol{\theta})$ in some sense (by choice of $\boldsymbol{\rho}$ ) one can achieve most accurate inference for the parameters of interest. For normal response trials with heteroscedastic outcomes, Wong and Zhu (2008) and Gwise, Zhu and Hu (2011) obtained $D A$-optimal designs that maximize efficiency for estimating treatment contrasts. Sverdlov and Rosenberger (2013a) give a comprehensive overview of various single- and multipleobjective optimal allocation designs that are available in the literature.

\subsection{Target Driven Randomization}

In general, the optimal allocation proportion vector $\boldsymbol{\rho}=\left(\rho_{1}, \ldots, \rho_{K}\right)$ derived as a solution to some optimization problem is a function of the distribution parameters $\boldsymbol{\theta}=\left(\boldsymbol{\theta}_{1}, \ldots, \boldsymbol{\theta}_{K}\right)$. In practice, one can construct a response-adaptive randomization design such that the sample allocation proportions sequentially converge to the chosen optimal allocation $\boldsymbol{\rho}(\boldsymbol{\theta})=$ $\left(\rho_{1}(\boldsymbol{\theta}), \ldots, \rho_{K}(\boldsymbol{\theta})\right)$. We shall assume that the target function $\rho(\boldsymbol{\theta})$ is a continuous function on the parameter space and twice differentiable at the true value of the parameter $\boldsymbol{\theta}=\left(\boldsymbol{\theta}_{1}, \ldots, \boldsymbol{\theta}_{K}\right)$. In general, one can use a smoothing mothod to modify the target function (c.f. Tymofyeyev, Rosenberger and Hu (2007)).

In the two-treatment trails with binary responses, the sample allocations $N_{n, 1} / n$ and $N_{n, 2} / n$ in both the RPW rule and DL rule sequentially converge to allocation proportion $\rho_{1}=q_{2} /\left(q_{1}+q_{2}\right)$ and $\rho_{2}=1-\rho_{1}$. However, the RPW and the DL rule can only target this specified allocation.

In general, given an allocation proportion vecotr $\boldsymbol{\rho}(\boldsymbol{\theta})$, if we apply the SEU model with

adding rules $\boldsymbol{D}_{m}=\beta \mathbf{1}^{\prime} \boldsymbol{\rho}\left(\widehat{\boldsymbol{\theta}}_{m}\right)(\beta>0)$, then we will have $\boldsymbol{N}_{n} / n \rightarrow \boldsymbol{\rho}(\boldsymbol{\theta})$ a.s. and the asymptotic normality given by (3.7). Here we always assume that $\widehat{\boldsymbol{\theta}}_{m}$ is the MLE of $\boldsymbol{\theta}$, or 
simply the sample means of the responses, based on the data from the previous $m$ trials. In a SEU model with adding rules $\boldsymbol{D}_{m}=\beta \mathbf{1}^{\prime} \boldsymbol{\rho}\left(\widehat{\boldsymbol{\theta}}_{m}\right)$, when the $(m+1)$-th patient is randomized, no matter what its response is, the urn is updated by adding additional $\beta \rho_{j}\left(\widehat{\boldsymbol{\theta}}_{m}\right)$ balls of type $j, j=1, \ldots, K$. The outcomes of treatments are only used to get the estimator $\widehat{\boldsymbol{\theta}}_{m}$.

Also, if we apply the IMU model with immigration rates $\boldsymbol{a}\left(\widehat{\boldsymbol{\theta}}_{m}\right)=\beta \boldsymbol{\rho}\left(\widehat{\boldsymbol{\theta}}_{m}\right)(\beta>0)$ and adding rules $\boldsymbol{D}_{m}=\operatorname{diag}(-1, \cdots,-1)$, then we will also have $\boldsymbol{N}_{n} / n \rightarrow \boldsymbol{\rho}(\boldsymbol{\theta})$ a.s. and the asymptotic normality given by (3.9). This procedure is the generalized drop-the-loser rule proposed by Zhang et al. (2007) and Sun, Cheung and Zhang (2007). In the GDL rule, when a treatment ball is drawn, it is always dropped. When an immigration ball is drawn, the ball is returned with additional $\beta \rho_{j}\left(\widehat{\boldsymbol{\theta}}_{m}\right)$ balls of type $j, j=1, \ldots, K$. The outcomes of treatments are only used to estimate the parameter $\boldsymbol{\theta}$ and get the current estimated immigration rates $\boldsymbol{\rho}\left(\widehat{\boldsymbol{\theta}}_{m}\right)$.

Another simple approach to construct a response-adaptive randomization design such that $\boldsymbol{N}_{n} / n \rightarrow \boldsymbol{\rho}(\boldsymbol{\theta})$ is defining the allocation probabilities of the $(m+1)$-th patient by

$$
p_{m+1, k}=\widehat{\rho}_{m, k}, \quad k=1, \ldots, K,
$$

where $\widehat{\boldsymbol{\rho}}_{m}=\left(\widehat{\rho}_{m, 1}, \ldots, \widehat{\rho}_{m, K}\right)$ with $\widehat{\rho}_{m, k}=\rho_{k}\left(\widehat{\boldsymbol{\theta}}_{m}\right)$ denotes the estimated target allocation. This is the sequential maximum likelihood procedure (SMLP) proposed by Melfi and Page (2000) and Melfi, Page, and Geraldes (2001).

A general allocation rule is defining the allocation probabilities as a function of both current sample allocation proportions and the estimated target allocation:

$$
p_{m+1, k}=g_{k}\left(\boldsymbol{N}_{m} / m, \widehat{\boldsymbol{\rho}}_{m}\right), \quad k=1, \ldots, K
$$

where $\boldsymbol{g}(\boldsymbol{x}, \boldsymbol{y})=\left(g_{1}(\boldsymbol{x}, \boldsymbol{y}), \ldots, g_{K}(\boldsymbol{x}, \boldsymbol{y})\right)\left(g_{k}(\boldsymbol{x}, \boldsymbol{y}) \geq 0\right.$ and $\left.\sum_{k=1}^{K} g_{k}(\boldsymbol{x}, \boldsymbol{y})=1\right)$ is called the allocation function. This is the doubly adaptive biased coin design proposed by $\mathrm{Hu}$ and Zhang (2004a), extending the work of Eisele (1994).

For a general function $\boldsymbol{g}$, the convergence of $\boldsymbol{N}_{n} / n$ is related to the stability of the following ordinary differential equation:

$$
\dot{\boldsymbol{x}}=\boldsymbol{x}-\boldsymbol{g}(\boldsymbol{x}, \boldsymbol{\rho}(\boldsymbol{\theta})), \text { with } \boldsymbol{x}=\boldsymbol{x}(s),
$$

where $\dot{\boldsymbol{x}}(s)$ is the derivative of $\boldsymbol{x}(s)$ (c.f. Zhang (2014)). If the function $\boldsymbol{g}$ is chosen such 
that $g_{k}(\boldsymbol{x}, \boldsymbol{y}) \leq \lambda\left(x_{k}-y_{k}\right)$ whenever $x_{k}>y_{k}$, where $0 \leq \lambda<1$, then

$$
\frac{\boldsymbol{N}_{n}}{n} \rightarrow \boldsymbol{\rho}(\boldsymbol{\theta}) \text { a.s. }
$$

This is proved by $\mathrm{Hu}$ and Zhang (2004a) and $\mathrm{Hu}$ et al. (2008), and they proposed the following allocation function:

$$
g_{k}(\boldsymbol{x}, \boldsymbol{y})=\frac{y_{k}\left(\frac{y_{k}}{x_{k}}\right)^{\gamma}}{\sum_{j=1}^{K} y_{j}\left(\frac{y_{j}}{x_{j}}\right)^{\gamma}}, \quad k=1, \ldots, K,
$$

where $\gamma \geq 0$ is user-defined parameter controlling the degree of randomness $(\gamma=0$ is almost completely randomized and $\gamma=\infty$ is almost deterministic procedure). The SMLP is a special case of DBCD with $\gamma=0$.

For considering the asymptotic normality, we note that

$$
\begin{gathered}
\widehat{\boldsymbol{\theta}}_{m, k}-\boldsymbol{\theta}_{k} \approx \frac{\sum_{j=1}^{m} X_{j, k} \boldsymbol{\eta}_{m, k}}{N_{m, k}} \approx \frac{\boldsymbol{B}_{k}(m) \boldsymbol{I}_{k}^{-1 / 2} \sqrt{\rho_{k}}}{m \rho_{k}}=\frac{\boldsymbol{B}_{k}(m) \boldsymbol{I}_{k}^{-1 / 2}}{m \sqrt{\rho_{k}}}, \\
\boldsymbol{M}_{n}=\sum_{j=1}^{m}\left(\boldsymbol{X}_{j}-\mathrm{E}\left[\boldsymbol{X}_{j} \mid \mathscr{F}_{j-1}\right]\right) \approx \boldsymbol{B}(t) \boldsymbol{\Sigma}_{1}^{1 / 2},
\end{gathered}
$$

where $\boldsymbol{\eta}_{m, k}$ is a function of the response $\boldsymbol{\xi}(m)$ with $\mathbf{E} \boldsymbol{\eta}_{m, k}=\mathbf{0}$ and $\operatorname{Var}\left(\boldsymbol{\eta}_{m, k}\right)=\boldsymbol{I}_{k}^{-1}$ with $\boldsymbol{I}_{k}=\boldsymbol{I}_{k}\left(\boldsymbol{\theta}_{k}\right)$ being the Fisher information for a single observation on treatment $k=1, \ldots, K, \boldsymbol{B}(t), \boldsymbol{B}_{1}(t), \ldots, \boldsymbol{B}_{K}(t)$ are independent multi-dimensional standard Browian motions, $\boldsymbol{\Sigma}_{1}=\operatorname{diag}(\boldsymbol{\rho})-\boldsymbol{\rho}^{\prime} \boldsymbol{\rho}$. Hereafter, for a symmetric and positive definite matrix $\boldsymbol{\Sigma}, \boldsymbol{\Sigma}^{1 / 2}$ is the symmetric matrix such that $\boldsymbol{\Sigma}^{1 / 2} \boldsymbol{\Sigma}^{1 / 2}=\boldsymbol{\Sigma}$, and $\boldsymbol{\Sigma}^{-1 / 2}$ is the inverse matrix of $\boldsymbol{\Sigma}^{1 / 2}$ satisfying $\boldsymbol{\Sigma}^{-1 / 2} \boldsymbol{\Sigma}^{-1 / 2}=\boldsymbol{\Sigma}^{-1}$. Write $\boldsymbol{W}(t)=\left(\boldsymbol{B}_{1}(t), \ldots, \boldsymbol{B}_{K}(t)\right)$ and $\boldsymbol{I}(\boldsymbol{\theta})=\operatorname{daig}\left(\rho_{1}(\boldsymbol{\theta}) \boldsymbol{I}_{1}, \ldots, \rho_{k}(\boldsymbol{\theta}) \boldsymbol{I}_{K}\right)$. Then

$$
\begin{aligned}
\widehat{\boldsymbol{\theta}}_{m}-\boldsymbol{\theta} & \approx \frac{\boldsymbol{W}(m) \boldsymbol{I}^{-1 / 2}(\boldsymbol{\theta})}{m}, \\
\Delta\left(\boldsymbol{N}_{m}-m \boldsymbol{v}\right) & \approx \Delta \boldsymbol{M}_{m, 1}+\left(\frac{\boldsymbol{N}_{m-1}}{m-1}-\boldsymbol{v}\right) \frac{\partial \boldsymbol{g}(\boldsymbol{\rho}, \boldsymbol{\rho})}{\partial \boldsymbol{x}}+\left(\widehat{\boldsymbol{\theta}}_{m-1}-\boldsymbol{\theta}\right) \frac{\partial \boldsymbol{\rho}(\boldsymbol{\theta})}{\partial \boldsymbol{\theta}} \frac{\partial \boldsymbol{g}(\boldsymbol{\rho}, \boldsymbol{\rho})}{\partial \boldsymbol{x}} .
\end{aligned}
$$

So

$$
\begin{gathered}
\boldsymbol{N}_{n}-n \boldsymbol{v} \approx \boldsymbol{G}(n) \text { with } \boldsymbol{G} \text { satisfying the SDE: } \\
d \boldsymbol{G}(t)=d \boldsymbol{B}(t) \boldsymbol{\Sigma}_{1}^{1 / 2}-\frac{\boldsymbol{G}(t)}{t} \frac{\partial \boldsymbol{g}(\boldsymbol{\rho}, \boldsymbol{\rho})}{\partial \boldsymbol{x}} d t+\frac{\boldsymbol{W}(t)}{t} \boldsymbol{I}^{-1 / 2}(\boldsymbol{\theta}) \frac{\partial \boldsymbol{\rho}(\boldsymbol{\theta})}{\partial \boldsymbol{\theta}} \frac{\partial \boldsymbol{g}(\boldsymbol{\rho}, \boldsymbol{\rho})}{\partial \boldsymbol{x}} d t
\end{gathered}
$$

Suppose the allocation function is chosen as in (4.1), then $\frac{\partial \boldsymbol{g}(\boldsymbol{\rho}, \boldsymbol{\rho})}{\partial \boldsymbol{x}}=-\gamma\left(\boldsymbol{I}-\boldsymbol{1}^{\prime} \boldsymbol{\rho}\right)$ and $\frac{\partial \boldsymbol{g}(\boldsymbol{\rho}, \boldsymbol{\rho})}{\partial \boldsymbol{y}}=(\gamma+1)\left(\boldsymbol{I}-\mathbf{1}^{\prime} \boldsymbol{\rho}\right)$. The SDE can be simplified to

$$
d \boldsymbol{G}(t)=d \boldsymbol{B}(t) \boldsymbol{\Sigma}_{1}^{1 / 2}-\gamma \frac{\boldsymbol{G}(t)}{t} d t+(\gamma+1) \frac{\boldsymbol{W}(t)}{t} \boldsymbol{I}^{-1 / 2}(\boldsymbol{\theta}) \frac{\partial \boldsymbol{\rho}(\boldsymbol{\theta})}{\partial \boldsymbol{\theta}} d t,
$$


which has a solution

$$
\boldsymbol{G}(t)=t^{-\gamma} \int_{0}^{t} x^{\gamma} d \boldsymbol{B}(x) \boldsymbol{\Sigma}_{1}^{1 / 2}+(\gamma+1) t^{-\gamma} \int_{0}^{t} x^{\gamma-1} \boldsymbol{W}(x) d x \boldsymbol{I}^{-1 / 2}(\boldsymbol{\theta}) \frac{\partial \boldsymbol{\rho}(\boldsymbol{\theta})}{\partial \boldsymbol{\theta}} .
$$

By deriving the variability of the Gaussian process, we conclude the asymptotic normality.

Theorem 4.1 Suppose the distributions $f_{1}\left(\cdot \mid \boldsymbol{\theta}_{1}\right), \ldots, f_{K}\left(\cdot \mid \boldsymbol{\theta}_{K}\right)$ of the outcomes of treatment $k=1, \ldots, K$ follow an exponential family. Let $\boldsymbol{g}(\boldsymbol{x}, \boldsymbol{y})$ be defined as (4.1). Then

$$
\begin{gathered}
\sqrt{n}\left(\widehat{\boldsymbol{\theta}}_{n}-\boldsymbol{\theta}\right) \stackrel{D}{\rightarrow} N\left(\mathbf{0}, \boldsymbol{I}^{-1}(\boldsymbol{\theta})\right), \\
\boldsymbol{N}_{n}-n \boldsymbol{\rho}=O(\sqrt{n \log \log n}) \text { a.s. and } \sqrt{n}\left(\frac{\boldsymbol{N}_{n}}{n}-\boldsymbol{\rho}\right) \stackrel{D}{\rightarrow} N\left(\mathbf{0}, \boldsymbol{\Sigma}_{\gamma}\right),
\end{gathered}
$$

where

$$
\boldsymbol{\Sigma}_{\gamma}=\boldsymbol{\Sigma}_{L B}+\frac{1}{1+2 \gamma}\left(\operatorname{diag}(\boldsymbol{\rho})-\boldsymbol{\rho}^{\prime} \boldsymbol{\rho}+\boldsymbol{\Sigma}_{L B}\right)
$$

with $\boldsymbol{\Sigma}_{L B}$ being defined as in (3.8).

We can also derive the asymptotic joint normality of $\widehat{\boldsymbol{\theta}}_{n}$ and $\frac{\boldsymbol{N}_{n}}{n}$, the asymptotic co-variance of them is $\operatorname{Cov}\left\{\widehat{\boldsymbol{\theta}}_{n}, \frac{\boldsymbol{N}_{n}}{n}\right\} \sim \frac{1}{n} \boldsymbol{I}(\boldsymbol{\theta}) \frac{\partial \boldsymbol{\rho}(\boldsymbol{\theta})}{\partial \boldsymbol{\theta}}$.

The asymptotic variability $\boldsymbol{\Sigma}_{\gamma}$ achieves its largest value when $\gamma=0$, and approaches its minimum value $\boldsymbol{\Sigma}_{L B}$ as $\gamma \rightarrow \infty$.

In the case of two-treatment trials with binary responses, the RPW rule, the DL rule, the SEU procedure with $\boldsymbol{D}_{m}=\mathbf{1}^{\prime} \boldsymbol{\rho}\left(\widehat{p}_{m, 1}, \widehat{p}_{m, 2}\right)$, the GDL rule, the SMLP and the DBCD can all applied to target the allocation $q_{2} /\left(q_{1}+q_{2}\right)$. The asymptotic variabilities of the sample allocation proportions $N_{n, 1} / n$ (after normalized by $\sqrt{n}$ ) are given in Table 1. It can be verified that the RPW rule always has the largest variability and the DL rule has the smallest variability. In fact,

$$
\sigma_{R P W}^{2}>\sigma_{S E U}^{2}>\sigma_{S M L P}^{2}>{ }_{\sigma_{D B C D}^{2}}^{\sigma_{G D L}^{2}}>\sigma_{D L}^{2},
$$

for all $0<q_{1}, q_{2}<1$.

The RPW rule and DL rule can only target the allocation $q_{2} /\left(q_{1}+q_{2}\right)$. The SEU design with $\boldsymbol{D}_{m}=\mathbf{1}^{\prime} \boldsymbol{\rho}\left(\widehat{\boldsymbol{\theta}}_{m}\right)$, the GDL, the SMLP and the DBCD can be used to target any desired allocation. Table 2 gives their asymptotic variabilities. 


\begin{tabular}{|c|c|c|c|}
\hline $\begin{array}{c}\text { Design } \\
\text { Variability }\left(\sigma^{2}\right)\end{array}$ & $\begin{array}{cl}\text { RPW } & \\
\frac{q_{1} q_{2}\left[3+2\left(p_{1}+p_{2}\right)\right]}{\left[2\left(q_{1}+q_{2}\right)-1\right]\left(q_{1}+q_{2}\right)^{2}}, & q_{q}+q_{2}>\frac{1}{2}, \\
\infty, & q_{1}+q_{2} \leq \frac{1}{2}\end{array}$ & $\begin{array}{c}\mathrm{DL} \\
\frac{q_{1} q_{2}\left(p_{1}+p_{2}\right)}{\left(q_{1}+q_{2}\right)^{3}}\end{array}$ & $\begin{array}{c}\text { SMLP } \\
\frac{q_{1} q_{2}\left(2+p_{1}+p_{2}\right)}{\left(q_{1}+q_{2}\right)^{3}}\end{array}$ \\
\hline Design & SEU & GDL & DBCD \\
\hline Variability $\left(\sigma^{2}\right)$ & $\frac{q_{1} q_{2}\left[2+5\left(p_{1}+p_{2}\right)\right]}{\left(q_{1}+q_{2}\right)^{3}}$ & $\frac{2 q_{1} q_{2}\left(p_{1}+p_{2}\right)}{\left(q_{1}+q_{2}\right)^{3}}$ & $\frac{q_{1} q_{2}\left[2+(1+2 \gamma)\left(p_{1}+p_{2}\right)\right]}{(1+2 \gamma)\left(q_{1}+q_{2}\right)^{3}}$ \\
\hline
\end{tabular}

Table 1: The asymptotic variabilities of RAR procedures with the same traget $\frac{q_{2}}{q_{1}+q_{2}}$.

\begin{tabular}{|c|c|c|c|c|}
\hline Design & SEU & GDL & SMLP & DBCD \\
Variability $(\boldsymbol{\Sigma})$ & $\boldsymbol{\Sigma}_{1}+6 \boldsymbol{\Sigma}_{L B}$ & $2 \boldsymbol{\Sigma}_{L B}$ & $\boldsymbol{\Sigma}_{1}+2 \boldsymbol{\Sigma}_{L B}$ & $\frac{1}{1+2 \gamma} \boldsymbol{\Sigma}_{1}+\frac{2+2 \gamma}{1+2 \gamma} \boldsymbol{\Sigma}_{L B}$ \\
\hline
\end{tabular}

Table 2: The asymptotic variabilities of RAR procedures with the same traget $\boldsymbol{\rho}(\boldsymbol{\theta})$.

\subsection{Variability and Efficiency}

For assessing theoretical operating characteristics of candidate designs, the first step is assessing the theoretical (limit) allocation proportions under certain criteria, which is the optimization problem as we discuss in subsection 4.1. The allocation proportion of the RPW rule is not optimal under usual criteria. For given a desired allocation $\boldsymbol{\rho}(\boldsymbol{\theta})$, the asymptotic variability of the sample allocation proportions $\boldsymbol{N}_{n} / n$ is perhaps the most important issue. The variability of sample allocation proportions can have a strong effect on power. This has been demonstrated by many simulation studies of Melfi and Page (1998) and Rosenberger et al. (2001), and theoretically by $\mathrm{Hu}$ and Rosenberger (2003), who show explicitly the relationship between the power of a test and the variability of the randomization procedure for a given allocation proportion. In the latter paper, the authors showed that the average power of a randomization procedure is a decreasing function of the variability of the procedure. Hu, Rosenberger and Zhang (2006) introduced asymptotically best response adaptive randomization procedures as ones that have the smallest variance of the allocation proportion among the procedures targeting the same allocation. These results allow a formal assessment of operating characteristics of various response-adaptive randomization 
designs. The following theorem of $\mathrm{Hu}$, Rosenberger and Zhang (2006) shows that for any given an allocation proportion, there is a lower bound of the asymptotic variability of the sample allocation proportions which converge to this given allocation proportion.

Theorem 4.2 Assume the following regularity conditions:

1. The parameter space $\boldsymbol{\Theta}$ of $\boldsymbol{\theta}$ is an open set;

2. The distributions of responses $f_{1}\left(\cdot \mid \boldsymbol{\theta}_{1}\right), \ldots, f_{K}\left(\cdot \mid \boldsymbol{\theta}_{1}\right)$ follow an exponential family;

3. For the limiting allocation proportion $\boldsymbol{\rho}(\boldsymbol{\theta})=\left(\rho_{1}(\boldsymbol{\theta}), \ldots, \rho_{K}(\boldsymbol{\theta})\right)$,

$$
\frac{N_{n, k}}{n} \rightarrow \rho_{k}(\boldsymbol{\theta}) \text { a.s. } k=1, \ldots, K
$$

4. For a positive definite matrix $\boldsymbol{V}(\boldsymbol{\theta})$,

$$
\sqrt{n}\left(\frac{N_{n}}{n}-\boldsymbol{\rho}(\boldsymbol{\theta})\right) \stackrel{D}{\rightarrow} N(\mathbf{0}, \boldsymbol{V}(\boldsymbol{\theta}))
$$

Then there exists a $\boldsymbol{\Theta}_{0} \subset \boldsymbol{\Theta}$ with Lebesgue measure 0 such that for every $\boldsymbol{\theta} \in \boldsymbol{\Theta} \backslash \boldsymbol{\Theta}_{0}$,

$$
\boldsymbol{V}(\boldsymbol{\theta}) \geq \boldsymbol{\Sigma}_{L B}
$$

where $\boldsymbol{\Sigma}_{L B}$ is defined by (3.8).

We refer to an adaptive design that attains the lower bound as asymptotic best (or efficient) for that particular allocation $\boldsymbol{\rho}(\boldsymbol{\theta})$. Table 3 gives the lower bounds of the asymptotic variabilities $\sigma_{L B}^{2}$ for the urn proportion $\frac{q_{2}}{q_{1}+q_{2}}$, the RSIHR proportion $\frac{\sqrt{p_{1}}}{\sqrt{p_{1}}+\sqrt{p_{2}}}$ and the Neyman proportion $\frac{\sqrt{p_{1} q_{1}}}{\sqrt{p_{1} q_{1}}+\sqrt{p_{2} q_{2}}}$ in a two-treatment clinical trial with binary responses.

\begin{tabular}{|c|c|c|}
\hline & $\rho_{1}(\boldsymbol{\theta})$ & $\sigma_{L B}^{2}$ \\
\hline Urn proportion & $\frac{q_{2}}{q_{1}+q_{2}}$ & $\frac{q_{1} q_{2}\left(p_{1}+p_{2}\right)}{\left(q_{1}+q_{2}\right)^{3}}$ \\
RSIHR proportion & $\frac{\sqrt{p_{1}}}{\sqrt{p_{1}}+\sqrt{p_{2}}}$ & $\frac{1}{4\left(\sqrt{p_{1}}+\sqrt{p_{2}}\right)^{3}}\left(\frac{p_{2} q_{1}}{\sqrt{p_{1}}}+\frac{p_{1} q_{2}}{\sqrt{p_{2}}}\right)$ \\
Neyman proportion & $\frac{\sqrt{p_{1} q_{1}}}{\sqrt{p_{1} q_{1}}+\sqrt{p_{2} q_{2}}}$ & $\frac{1}{4\left(\sqrt{p_{1} q_{1}}+\sqrt{p_{2} q_{2}}\right)^{3}}\left(\frac{p_{2} q_{2}\left(1-2 p_{1}\right)^{2}}{\sqrt{p_{1} q_{1}}}+\frac{p_{1} q_{1}\left(1-2 p_{2}\right)^{2}}{\sqrt{p_{2} q_{2}}}\right)$ \\
\hline
\end{tabular}

Table 3: Lower bounds of RAR procedures for several allocation proportions. 
For the case of two-treatment trials with binary responses, among the randomization procedures as given in the last subsection which target the same allocation $q_{2} /\left(q_{1}+q_{2}\right)$, the RPW rule has the largest variability which is faraway from the lower bound, and the DL rule is an asymptotically best response adaptive randomization procedure. For a general allocation proportion $\boldsymbol{\rho}(\boldsymbol{\theta})$, only the DBCD can approach an asymptotically best response adaptive randomization procedure as $\gamma \rightarrow \infty$ (Table 2).

\subsection{Efficient RAR Designs}

$\mathrm{Hu}$, Zhang and He (2009) proposed a class of efficient response adaptive randomization designs, which are fully randomized, can target any allocation (under mild regularity conditions), and are asymptotically best. The ERADE was proposed for comparing two treatments. After $m$ assignments, we let $\widehat{\boldsymbol{\theta}}_{m}=\left(\widehat{\boldsymbol{\theta}}_{m, 1}, \widehat{\boldsymbol{\theta}}_{m, 2}\right)$ be the MLE of the parameter $\boldsymbol{\theta}=\left(\boldsymbol{\theta}_{1}, \boldsymbol{\theta}_{2}\right)$. The probability of assigning the $(m+1)$-th patient to treatment 1 is defined by

$$
p_{m+1,1}= \begin{cases}\alpha \rho_{1}\left(\widehat{\boldsymbol{\theta}}_{m}\right), & \text { if } \frac{N_{m}}{m}>\rho_{1}\left(\widehat{\boldsymbol{\theta}}_{m}\right), \\ \rho_{1}\left(\widehat{\boldsymbol{\theta}}_{m}\right), & \text { if } \frac{N_{m}}{m}=\rho_{1}\left(\widehat{\boldsymbol{\theta}}_{m}\right), \\ 1-\alpha\left(1-\rho_{1}\left(\widehat{\boldsymbol{\theta}}_{m}\right)\right), & \text { if } \frac{N_{m}}{m}<\rho_{1}\left(\widehat{\boldsymbol{\theta}}_{m}\right),\end{cases}
$$

where $0 \leq \alpha<1$ is a pre-specified constant. When $\rho(\boldsymbol{\theta}) \equiv 1 / 2$, the procedure reduces to the famous Efron's biased coin design (Efron (1971)). The constant $\alpha$ is related to the randomness of the design. When $\alpha=1$, the procedure reduces to the SMLP. When $\alpha$ is smaller, the ERADE is more deterministic and could have smaller variability. Hu, Zhang and He (2009) recommend choosing $\alpha$ between 0.4 and 0.7 .

Theorem 4.3 Suppose the distributions $f_{1}\left(\cdot \mid \boldsymbol{\theta}_{1}\right)$ and $f_{2}\left(\cdot \mid \boldsymbol{\theta}_{2}\right)$ of the responses follow an exponential family. Then

$$
\begin{gathered}
N_{n, 1}-n \rho_{1}=O(\sqrt{n \log \log n}) \text { a.s. and } \\
\max _{m \leq n}\left|N_{m, 1}-m \rho_{1}\left(\widehat{\boldsymbol{\theta}}_{m}\right)\right|=o(\sqrt{n}) \text { in probability, }
\end{gathered}
$$

where $\rho_{1}=\rho_{1}(\boldsymbol{\theta})$. In particular,

$$
\sqrt{n}\left(\widehat{\boldsymbol{\theta}}_{n}-\boldsymbol{\theta}, \frac{N_{n, 1}}{n}-\rho_{1}\right) \stackrel{D}{\rightarrow} N(\mathbf{0}, \boldsymbol{\Lambda}) \quad \text { with }
$$




$$
\boldsymbol{\Lambda}=\left(\begin{array}{cc}
\boldsymbol{I}^{-1}(\boldsymbol{\theta}) & \boldsymbol{I}^{-1}(\boldsymbol{\theta}) \frac{\partial \rho_{1}(\boldsymbol{\theta})}{\partial \boldsymbol{\theta}} \\
\left(\frac{\partial \rho_{1}(\boldsymbol{\theta})}{\partial \boldsymbol{\theta}}\right)^{\prime} \boldsymbol{I}^{-1}(\boldsymbol{\theta}) & \sigma_{L B}^{2}
\end{array}\right), \quad \sigma_{L B}^{2}=\left(\frac{\partial \rho_{1}(\boldsymbol{\theta})}{\partial \boldsymbol{\theta}}\right)^{\prime} \boldsymbol{I}^{-1}(\boldsymbol{\theta}) \frac{\partial \rho_{1}(\boldsymbol{\theta})}{\partial \boldsymbol{\theta}}
$$

Under the situation where efficiency is critically important, in theory, the ERADE should be the best choice in all the response-adaptive randomization procedures. The simulation evidence that the ERADE outperforms other procedures in most cases can be found in $\mathrm{Hu}$, Zhang and He (2009), Flournoy, Haines and Rosenberger (2014) etc. When the responses are binary and the desired proportion is $q_{2} /\left(q_{1}+q_{2}\right)$, the DL rule is also an efficient responseadaptive randomization procedure. However, simulations shows that the DL rule produces an allocation that is suboptimal with respect to power. Its finite-sample variances are much smaller than the corresponding asymptotic variances, but when $p_{1}$ and $p_{2}$ are large and different, the DL rule does not converge to the target allocation proportion as fast as other procedures (c.f. Hu, Zhang and $\mathrm{Hu}(2009))$. Similar simulation evidence is also found for the ERADE under some situations when the sample size is small. It sometimes does not converge to the target allocation proportion as fast as the DBCD does, though its finitesample variances are always small. The main reason for such a phenomenon is possibly that the allocation probabilities in the DL rule and the the ERADE are not stable, they always jump from one value to another. A continuous allocation function can make the allocation probabilities stable and speed up the convergence of the sample allocation proportions.

Very recently, Zhang et al. (2014b) proposed a new ERADE for multi-treatment trials by defining a continuous allocation function. Let $\psi(x)$ be a weight function given by

$$
\psi(x)=1+\sqrt{\left(x^{2 \gamma}-1\right) \vee 0}, \quad x \geq 0 .
$$

Define the allocation function $\boldsymbol{g}(\boldsymbol{x}, \boldsymbol{y})=\left(g_{1}(\boldsymbol{x}, \boldsymbol{y}), \ldots, g_{K}(\boldsymbol{x}, \boldsymbol{y})\right)$ by

$$
g_{k}(\boldsymbol{x}, \boldsymbol{y})=\frac{y_{k} \psi\left(\frac{y_{k}}{x_{k}}\right)}{\sum_{j=1}^{K} y_{j} \psi\left(\frac{y_{j}}{x_{j}}\right)}, \quad k=1, \ldots, K .
$$

After $m$ assignments, the probability of assigning the $(m+1)$-th patient to treatment $k$ is defined by

$$
p_{m+1, k}=g_{k}\left(\frac{\boldsymbol{N}_{m}}{m}, \widehat{\boldsymbol{\rho}}_{m}\right) \text { with } \widehat{\boldsymbol{\rho}}_{m}=\rho\left(\widehat{\boldsymbol{\theta}}_{m}\right), \quad k=1, \ldots, K
$$


Theorem 4.4 Suppose the distributions $f_{1}\left(\cdot \mid \boldsymbol{\theta}_{1}\right), \ldots, f_{K}\left(\cdot \mid \boldsymbol{\theta}_{K}\right)$ of the responses follow an exponential family. Then $p_{m+1, k} \rightarrow \rho_{k}, k=1, \ldots, K$,

$$
\begin{gathered}
\boldsymbol{N}_{n}-n \boldsymbol{\rho}=O(\sqrt{n \log \log n}) \text { a.s. and } \\
\max _{m \leq n}\left\|\boldsymbol{N}_{m}-m \boldsymbol{\rho}\left(\widehat{\boldsymbol{\theta}}_{m}\right)\right\|=o(\sqrt{n}) \text { in probability, }
\end{gathered}
$$

where $\boldsymbol{\rho}=\left(\rho_{1}, \ldots, \rho_{K}\right)=\boldsymbol{\rho}(\boldsymbol{\theta})$. Further there is a multi-dimensional standard Brownian motion $\boldsymbol{W}(t)$ such that

$$
\begin{gathered}
n\left(\widehat{\boldsymbol{\theta}}_{n}-\boldsymbol{\theta}\right)=\boldsymbol{W}(n) \boldsymbol{I}^{-1 / 2}(\boldsymbol{\theta})+o(\sqrt{n}) \text { a.s. and } \\
\max _{m \leq n}\left\|\boldsymbol{N}_{m}-m \boldsymbol{\rho}-\boldsymbol{W}(m) \boldsymbol{I}^{-1 / 2}(\boldsymbol{\theta}) \frac{\partial \boldsymbol{\rho}(\boldsymbol{\theta})}{\partial \boldsymbol{\theta}}\right\|=o(\sqrt{n}) \text { in probability. }
\end{gathered}
$$

In particular,

$$
\begin{aligned}
& \sqrt{n}\left(\widehat{\boldsymbol{\theta}}_{n}-\boldsymbol{\theta}, \frac{\boldsymbol{N}_{n}}{n}-\boldsymbol{\rho}\right) \stackrel{D}{\rightarrow} N(\mathbf{0}, \boldsymbol{\Lambda}) \quad \text { with } \\
& \boldsymbol{\Lambda}=\left(\begin{array}{cc}
\boldsymbol{I}^{-1}(\boldsymbol{\theta}) & \boldsymbol{I}^{-1}(\boldsymbol{\theta}) \frac{\partial \boldsymbol{\rho}(\boldsymbol{\theta})}{\partial \boldsymbol{\theta}} \\
\left(\frac{\partial \boldsymbol{\rho}(\boldsymbol{\theta})}{\partial \boldsymbol{\theta}}\right)^{\prime} \boldsymbol{I}(\boldsymbol{\theta}) & \boldsymbol{\Sigma}_{L B}
\end{array}\right), \quad \boldsymbol{\Sigma}_{L B}=\left(\frac{\partial \boldsymbol{\rho}(\boldsymbol{\theta})}{\partial \boldsymbol{\theta}}\right)^{\prime} \boldsymbol{I}^{-1}(\boldsymbol{\theta}) \frac{\partial \boldsymbol{\rho}(\boldsymbol{\theta})}{\partial \boldsymbol{\theta}} .
\end{aligned}
$$

\section{Selection Bias and Lack of Randomness}

If the experimenter can predict the next assignment, he may consciously or unconsciously bias the experiment as to what treatment particular types of subjects should receive. Randomization is used for neutralizing such bias in clinical trials. A natural measure of the selection bias of a sequential design is the expected percentage of correct guesses the experimenter can make if he guesses optimally (c.f., Efron (1971)). Let $J_{m}=1$ if the $m$ th assignment is guessed correctly, and $J_{m}=0$ otherwise. The expected proportion of correct guesses is the expected value of $\frac{1}{n} \sum_{m=1}^{n} J_{m}$. So selection bias of the design is defined by

$$
S B_{n}=\mathrm{E}\left[\frac{1}{n} \sum_{m=1}^{n} J_{m}\right]=\frac{1}{n} \sum_{m=1}^{n} \mathrm{P}\left(J_{m}=1\right) .
$$

It is obvious that, for a complete randomization, in which each patient is assigned to each one of the $K$ treatments with the same probability $1 / K$, the selection bias is $S B_{n}=1 / K$ which is the smallest value of the selection biases of all randomization procedure in a trail with $K$ treatments. The selection bias can be regarded as a measure of lack of randomness. 
In a response-adaptive randomization procedure, the optimal guessing strategy is to guess treatment $k$ for which $p_{m+1, k}=\max _{j} p_{m+1, j}$. So

$$
P\left(J_{m+1}=1\right)=\mathrm{E}\left[\max _{k} p_{m+1, k}\right]
$$

It is obvious that $\frac{1}{K} \leq S B_{n} \leq 1$. When the allocation is not balance, the optimal value $1 / K$ can not be attained. Zhang et al. (2014b) gives the minimum value of the asymptotic selection bias.

Theorem 5.1 For any adaptive design, if $\boldsymbol{N}_{n} / n \rightarrow \boldsymbol{\rho}(\boldsymbol{\Theta})$ in probability, then

$$
\liminf _{n \rightarrow \infty} S B_{n} \geq \max _{k} \rho_{k}(\boldsymbol{\Theta})
$$

Further, if $p_{m+1, k} \rightarrow \rho_{k}(\boldsymbol{\theta})$ in probability, $k=1, \ldots, K$, then

$$
S B=: \lim _{n \rightarrow \infty} S B_{n}=\max _{k} \rho_{k}(\boldsymbol{\Theta})
$$

According to this theorem, $\max _{k} \rho_{k}(\boldsymbol{\Theta})$ is the minimum value of the asymptotic selection bias with a target allocation proportion $\boldsymbol{\rho}(\boldsymbol{\Theta})$. And, if the allocation probabilities converge to the target allocation proportion, then the design attains the lower bound of the selection bias. As a conclusion, the GPU, the SEU, the DBCD, the SMLP (as a special case of DBCD) and the new ERADE of Zhang et al. (2014b) attains the lower bound of the selection bias. However, the asymptotic selection bias $S B$ of the $\mathrm{Hu}$, Zhang and $\mathrm{He}(2009)$ procedure is a monotone function of the parameter $\alpha$, which coincides the intuition via the definition of the allocation function.

Theorem 5.2 Consider the procedure of Hu, Zhang and He (2009) for two-treatment clinical trials. Suppose that $P\left(\rho_{1}\left(\widehat{\boldsymbol{\Theta}}_{m}\right)=v\right)=0$ for any rational $v \in[0,1]$. Then we have

$$
S B= \begin{cases}1-2 \alpha \rho_{1} \rho_{2}, & \text { if } \rho_{1} \vee \rho_{2} \leq \frac{1}{2 \alpha}, \\ \rho_{1} \vee \rho_{2}, & \text { if } \rho_{1} \vee \rho_{2} \geq \frac{1}{2 \alpha}\end{cases}
$$

It turns out that only the new ERADE of Zhang et al. (2014b) achieves both the lower bound of asymptotic variability and the lower bound of the selection bias.

Recall that for a sequence $Y_{1}, Y_{2}, \ldots, Y_{n}$ of random variables taking values 0 and 1 with mean $p$, they are completely random if and only if $\mathrm{E}\left[Y_{i} \mid \mathscr{F}_{i-1}\right]=p, i=1, \ldots, n$. So

$$
\frac{1}{n} \sum_{i=1}^{n} \mathrm{E}\left|\mathrm{E}\left[Y_{i} \mid \mathscr{F}_{i-1}\right]-p\right|,
$$


can be regarded as a measure of the lack of randomness of this sequence. Therefore, for a response-adaptive randomization procedure, we can define another measure of the lack of randomness by

$$
M L R_{n}=\frac{1}{n} \sum_{m=1}^{n} \frac{1}{K} \sum_{k=1}^{K} \mathrm{E}\left|p_{m, k}-\rho_{k}\right| .
$$

It is obvious that if $p_{m, k} \rightarrow \rho_{k}$ in probability, $k=1, \ldots, K$, then

$$
M L R=: \lim _{n \rightarrow \infty} M L R_{n}=0 .
$$

So, for the GPU, the SEU, the DBCD and the new ERADE of Zhang et al. (2014b), $M L R=0$. For the procedure of $\mathrm{Hu}$, Zhang and He (2009), $M L R=2(1-\alpha) \rho_{1} \rho_{2}$ is a also a decreasing function of $\alpha$. The conclusion coincides with that for $S B$.

For further considering the degree of the lack of randomness of the designs with $M L R=$ 0 , we take the case of $K=2$ as example. Recall that in the RPW rule,

$$
p_{m+1,1}=\frac{Y_{m, 1}}{m} \stackrel{D}{\sim} N\left(\rho_{1}, \frac{\tilde{\sigma}_{R P W}^{2}}{m}\right), \quad \tilde{\sigma}_{R P W}^{2}=\frac{q_{1} q_{2}}{\left(2\left(q_{1}+q_{2}\right)-1\right)\left(q_{1}+q_{2}\right)^{2}},
$$

when $q_{1}+q_{2}>1 / 2$, and $\widetilde{\sigma}_{R P W}^{2}=\infty$ when $q_{1}+q_{2} \leq 1 / 2$. So,

$$
\lim _{n \rightarrow \infty} \sqrt{n}\left(M L R_{n}\right)=\lim _{n \rightarrow \infty} \frac{1}{\sqrt{n}} \sum_{m=1}^{n} \frac{\sqrt{2 / \pi} \widetilde{\sigma}_{R P W}}{m^{1 / 2}}=\sqrt{8 / \pi} \widetilde{\sigma}_{R P W},
$$

when $q_{1}+q_{2}>1 / 2$, and $\lim _{n \rightarrow \infty} \sqrt{n}\left(M L R_{n}\right)=\infty$ when $q_{1}+q_{2} \leq 1 / 2$. Similarly, for the DBCD,

$$
\lim _{n \rightarrow \infty} \sqrt{n}\left(M L R_{n}\right)=\sqrt{8 / \pi} \widetilde{\sigma}_{D B C D}, \quad \widetilde{\sigma}_{D B C D}^{2}=\frac{\gamma^{2} \rho_{1} \rho_{2}+(1+\gamma)^{2} \sigma_{L B}^{2}}{1+2 \gamma}
$$

Because $\widetilde{\sigma}_{D B C D}^{2}$ is an increasing function of $\gamma$, the large is $\gamma$, the more is the lack of randomness of the design. When $\gamma=0$, the procedure is the most random one in the family of DBCDs and $\lim _{n \rightarrow \infty} \sqrt{n}\left(M L R_{n}\right)$ takes the smallest value $\sqrt{8 / \pi} \sigma_{L B}$. This coincides the intuition via the definition of the allocation function. It is easily to show that in the binary response case with target allocation $q_{2} /\left(q_{1}+q_{2}\right), \widetilde{\sigma}_{R P W}$ is also greater than $\sigma_{L B}^{2}$. We conjecture that in the class of all adaptive randomization procedures with a same allocation target, the DBCD with $\gamma=0$ (i.e. the SMLP) is the most random one. When $\gamma=0$, the allocation probability is $\rho_{1}\left(\widehat{\boldsymbol{\theta}}_{m}\right)$ which is an asymptotically best estimator of $\rho_{1}(\boldsymbol{\theta})$, and so the adaptive randomization procedure is locally efficient.

For the new ERADE of Zhang et al. (2014b), we have $\lim _{n \rightarrow \infty} \sqrt{n}\left(M L R_{n}\right)=\infty$. 
Hence, the efficiency and the randomness are always two conflicting features of adaptive randomization designs. However, it is surprising that the variability of the RPW rule is much higher than other designs including the DBCD, and at the same time, the RPW rule is much less random than the DBCD when $p_{1}+p_{2}$ is close to or exceeds 1.5. The value of $S B$ and $M L R$ for the DL rule, the GDL rule and the IMU design is an open problem. It is very possibly a positive constant depending the initial value of the urn composition.

\section{Survival and Delayed Responses}

In the framework of response-adaptive randomization designs discussed above, the outcomes of the treatments are assumed to be complete and available immediately. In practices, many clinical trials have time-to-event outcomes and the outcomes may be censored. The problem of handling survival responses in response-adaptive randomization designs have been studied by Rosenberger and Seshaiyer (1997), Zhang and Rosenberger (2007), Sverdlov Ryeznik and Wong (2014) etc. For applying the response-adaptive randomization methodology to survival trials, the first problem is how to derive the desired allocation under reasonable optimization criteria. Several meaningful optimization methods have been proposed by Zhang and Rosenberger (2007) and Sverdlov Ryeznik and Wong (2014) in the framework of parameter survival models. The other problem is that, when the outcomes are time-toevent, they are usually delayed and will not be observed before the next step of the sequential procedure is carried out. The delay time is usually the observed time or the censoring time. The estimation of the parameters and the updatie of the urn composition (when using urn models), can only be processed according to observed responses. The effect of the delay of treatment results is fist studied in theory by Bai, Hu and Rosenberger (2002) for the urn compositions in GPU designs. After that, Hu and Zhang (2004b), Zhang et al. (2007), Sun,

Cheung and Zhang (2007) and $\mathrm{Hu}$ et al. (2008) has showed that the delay machine does not effect the asymptotic properties of the sample allocation proportions for many adaptive designs if the delay degree decays with a power rate. The basic reason is that the total delayed responses is a high order infinitesimal of square root of the sample size when the delay degree decays with a power rate.

To describe the delay machine, we let $t_{m}$ be the entry time of the $m$-th patient, where 
$t_{m}$ is an increasing sequence of random variables. Assume that $\left\{t_{m+1}-t_{m}\right\}$ is a sequence of independent random variables. The response time of the $m$-th patient on treatment $k$ is denoted by $r_{m}(k)$. Suppose $\left\{r_{m}(k) ; m \geq 1\right\}$ is a sequence of independent random variables, $k=1, \ldots, K$. Further, assume that $\left\{t_{m+1}-t_{m}, r_{m}(k) ; k=1, \ldots, K, m \geq n\right\}$ is independent of the assignments $\boldsymbol{X}_{1}, \ldots, \boldsymbol{X}_{n}$.

Assumption 6.1 Let $\delta_{k}(m, \ell)=I\left\{r_{m}(k) \geq t_{m+\ell}-t_{m}\right\}$ be an indicator function that takes the value 1 if the outcome of the $m$-th patient on treatment $k$ occurs after at least another $\ell$ patients arrive at the trials, and 0 otherwise. Suppose for some constans $C>0$ and $\beta \geq 2$,

$$
\mu_{k}(m, \ell)=P\left(\delta_{k}(m, \ell)=1\right) \leq C \ell^{-\beta} \text {, for all } m, \ell, k \text {. }
$$

This assumption is widely satisfied. A practical approach is to assume that the entry mechanism generates a Poisson process and the delay time has an exponential distribution in which both $\left\{r_{m}(k)\right\}$ and $\left\{t_{m+1}-t_{m}\right\}$ are sequences of i.i.d. exponential random variables with means $\lambda_{k}>0$ and $\lambda_{0}>0$, respectively. This approach is common in clinical studies and the probability $\mu_{k}(m, \ell)$ is $\left(\lambda_{k} /\left(\lambda_{0}+\lambda_{k}\right)\right)^{\ell}$.

Let $S_{m, k}^{o b s}$ (resp. $N_{m, k}^{o b s}$ ) be the summation (resp. the number) of the outcomes on treatment $k$ observed prior to the $(m+1)$-th assignment, and $S_{m, k}$ (resp. $\left.N_{m, k}\right)$ be the summation (resp. the number) of all the outcomes of those being assigned to treatment $k$ in the first $m$ patients, $k=1, \ldots, K$. The total delayed responses are then $S_{m, k}-S_{m, k}^{o b s}, k=1, \ldots, K$. Then we have the following theorem.

Theorem 6.1 Suppose Assumption 6.1 is satisfied, and the responses on each treatment are i.i.d. random variables having finite $(2+\delta)$-th moments. Then for some $0<\delta_{0}<\frac{1}{2}-\frac{1}{2+\delta}$, we have

$$
S_{m, k}-S_{m, k}^{o b s}=o\left(n^{1 / 2-\delta_{0}}\right) \text { a.s. }
$$

and $N_{m, k}-N_{m, k}^{o b s}=o\left(n^{1 / 2-\delta_{0}}\right)$ a.s.

\section{Other RAR Designs and Concluding Remarks}

Besides the response-adaptive randomization designs disused in this paper, many other procedures have been proposed in literature. For example, Thompson (1933) described a 
Bayesian procedure to compute the probability that one treatment is better than another with binary responses. Under a uniform prior distribution, the procedure yields the following formula. Given sufficient statistics $\left(S_{m, 1}, S_{m, 2}, N_{m, 1}, N_{m, 2}\right)$ after $m$ patients, where $S_{m, 1}$, $S_{m, 2}$ are the numbers of successes of treatment 1 and 2 respectively, then

$$
\widehat{P}\left(p_{1}>p_{2}\right)=\frac{\sum_{a=0}^{S_{m, 1}}\left(\begin{array}{c}
S_{m, 1}+S_{m, 2}-a \\
S_{m, 2}
\end{array}\right)\left(\begin{array}{c}
N_{m, 1}-S_{m, 1}+N_{m, 2}-S_{m, 2} \\
N_{m, 2}-S_{m, 2}
\end{array}\right)}{\left(\begin{array}{c}
n+2 \\
N_{m, 2}+1
\end{array}\right)} .
$$

Thall and Wathen (2007) reformulated this procedure by proposing the following responseadaptive procedure:

$$
p_{m+1, k}=\frac{\left[\widehat{P}\left(p_{1}>p_{2}\right)\right]^{c}}{\left[\widehat{P}\left(p_{1}>p_{2}\right)\right]^{c}+\left[1-\widehat{P}\left(p_{1}>p_{2}\right)\right]^{c}} .
$$

They suggested selecting $c=\left(N_{m, 1}+N_{m, 2}\right) /(2 n)$ and updating $c$ adaptively to reduce the root MSE of the procedure.

Various designs are usually proposed in different views. Note that a design is "good" under one criterion may be not "good" under another criterion. A graphical comparison of response-adaptive randomization procedures can be found in Flournoy, Haines and Roenberger (2014).

In this paper, we give an overview of important response-adaptive randomization designs and their asymptotic theory. In practice, a successful application of a response-adaptive randomization design in a clinical trial may depend on at least three essentials. The first one is a good adaptive procedure. We suggest that the procedure should be fully randomized, have small variabilities and converges fast to an allocation which is optimal under a given optimization criterion. The main drawback of the RPW is that it has high variability. Also, its allocation proportion is not an optimal allocation under opportune optimization criteria. The second essential is a good estimation method to collect the information from the data on time. The sufficient statics and MLE are usually suggested. When the sample size is small, the Bayesian method is a reasonable candidate. Other estimators such as weighted likelihood estimators, robust estimators can be also used. The third essential is a not-worse start of the procedure. The performance of the RPW and urn models depend heavily on the initial composition of the urn, especially when the sample size is not large. In a pediatric trial of extracorporeal membrane oxygenation (ECMO; Bartlett et al., 1985), the RPW rule with 2 initial balls, 1 of each type, was used to randomize the patients. After several initial 
assignments in which the balls added were all of the ECMO type, the urn composition became very extreme so that the conventional therapy has very little chance to be selected. If the RPW rule with 10 initial balls, 5 of each type, had been used, maybe the story would have been different. The convergence of the urn proportions and the sample allocation proportions for the RPW rule is very slow. The DBCD and the ERADE converge much faster than the urn models. But also the initial estimator of the parameters may have some effects on their operating characteristics, especially when the sample size is very small. A relatively accurate initial estimator may improve the performance of the design. If there is not enough information to get a good estimator at the initial several steps, the estimator or the allocation probabilities are suggested to be modified such that the randomization is close to the restricted randomization until a rwasonable quality estimator is available. For details of the discuss about the applications of response-adaptive randomization designs, one can refer to Rosenberger, Sverdlov and Hu (2012) and Sverdlov and Rosenberger (2013b).

\section{REFERENCES}

Bai, Z. D., Hu, F. (2005). Asymptotics in randomized urn models. Annals of Applied Probability, 15: $914-940$.

Bai, Z. D., Hu, F., Rosenberger, W. F. (2002). Asymptotic properties of adaptive designs for clinical trials with delayed response. Annals of Statistics, 30: 122-139.

Bai, Z. D., Hu, F. and Zhang, L.-X. (2002). The Gaussian approximation theorems for urn models and their applications. Annals of Applied Probability, 12: 1149-1173.

Baldi Antognini, A., Giovagnoli, A. (2010). Compound optimal allocation for individual and collective ethics in binary clinical trials. Biometrika, 97: 935-946.

Bartlett, R. H., Rolloff, D. W., Cornell, R. G., Andrews, A. F., Dillon, P. W., Zwischenberger, J. B. (1985). Extracorporeal circulation in neonatal repiratory failure: a prospective randomized trial. Pediatrics, 76: 479-487.

Biswas, A., Bhattacharya, R. (2009). Optimal response-adaptive designs for normal responses. Biometrical Journal, 51: 193-202.

Biswas, A., Bhattacharya, R. (2010). An optimal response-adaptive design with dual constraints. Statistics and Probability Letters, 80: 177-185.

Biswas, A., Bhattacharya, R. (2011). Optimal response-adaptive allocation designs in phase III clinical trials: Incorporating ethics in optimality. Statistics and Probability Letters, 81: 11551160.

Biswas, A., Mandal (2004). Optimal adaptive designs in pase III clinical trials for continuuos responses with covariates. In: Bucchianico, A. Di., Lauter, H., Wynn, H. P., eds. mODa 7-Advances in Model-Oriented Design and Analysis. Heidelberg: Physica-Verlag, pp. 51-58.

Eisele, J. R. (1994). The doubly-adaptive biased coin design for sequential clinical trials. Journal of Statistical Planning and Inference, 38: 249-262. 
Efron, B. (1971). Forcing a sequential experiment to be balanced. Biometrika, 58: 403-417.

Gwise, T., Hu, J., Hu, F. (2008). Optimal biased coins for two-arm clinical trials. Statistics and its Interface, 1: 125-135.

Flournoy, N., Haines, L. M., Rosenberger, W. F. (2013). A graphical comparison of response-adaptive randomization procedures. Statistics in Biopharmaceutical Research, 5: 126-141.

Gwise, T., Zhu, J., Hu, F. (2011). An optimal response adaptive biased coin design with $k$ heteroscedastic treatments. Journal of Statistical Planning and Inference, 141: 235-242.

Hu, F., Rosenberger, W. F. (2003). Optimality, variability, power: Evaluating responseadaptive randomization procedures for treatment comparisons. Journal of the American Statistical Association, 98: 671-678.

Hu, F., Rosenberger, W. F. (2006). The Theory of Response-Adaptive Randomization in Clinical Trials. New York: Wiley.

Hu, F., Rosenberger, W. F., Zhang, L.-X. (2006). Asymptotically best response- adaptive randomization procedures. Journal of Statistical Planning and Inference, 136: 1911-1922.

Hu, F., Zhang, L.-X. (2004a). Asymptotic properties of doubly-adaptive biased coin designs for multitreatment clinical trials. Annals of Statistics, 32: 268-301.

Hu, F., Zhang, L.-X. (2004b). Asymptotic normality of adaptive designs with delayed response.Bernoulli,10: 447-463.

Hu, F., Zhang, L.-X., Cheung, S. H., Chan, W. S. (2008). Doubly-adaptive biased coin designs with delayed responses. Canadian Journal of Statistics, 36: 541-559.

Hu, F., Zhang, L.-X., He, X. (2009). Efficient randomized-adaptive designs. Annals of Statistics, 37: 2543-2560.

Ivanova, A. V. (2003). A play-the-winner type urn model with reduced variability. Metrika, 58: $1-14$.

Ivanova, A. V. (2006). Urn designs with immigration: Useful connection with continuous time stochastic processes. Journal of Statistical Planning and Inference, 136: 1836-1844.

Ivanova, A. V., Rosenberger, W. F. (2001). Adaptive designs for clinical trials with highly successful treatments. Drug Information Journal, 35: 1087-1093.

Jennison, C., Turnbull, B. W. (2000). Group Sequential Methods with Applications to Clinical Trials. Boca Raton, FL: Chapman and Hall/CRC.

Jeon, Y., Hu, F. (2010). Optimal adaptive designs for binary response trials with three treatments. Statistics in Biopharmaceutical Research, 2(3): 310-318.

Li, W., Durham, S. D. and Flournoy, N. (1996). Randomized Pólya urn designs. Proceedings of the Biometric Section of the Statistical Association: 166-170.

May, C. and Flournoy, N. (2009). Asymptotics in response-adaptive designs generated by a two-color, randomly reinforced urn. Annals of Statistics, 37(2): 1058-1078.

Melfi, V. and Page, C. (1998). Variability in adaptive designs for estimation of success probabilities. In New Developments and Applications in Experimental Design (N. Flournoy, W. F. Rosenberger and W. K. Wong, eds.) 106-114. IMS, Hayward, CA.

Melfi, V. and Page, C. (2000). Estimation after adaptive allocation. Journal of Statistical Planning and Inference, 87: 353-363.

Melfi, V. and Page, C. and Geraldes, M. (2001). An adaptive randomized design with application to estimation. Canadian Journal of Statistics, 29: 107-116. 
Robbins, H. (1952). Some aspects of the sequential design of experiments. Bulletin of the American Mathematical Society, 58: 527-535.

Rosenberger, W. F. (2002). Randomized urn models and sequential design. Sequential Analysis, 21:1-41 (with discussion).

Rosenberger, W. F., Hu, F. (2004). Maximizing power and minimizing treatment failures in clinical trials. Clinical Trials, 1: 141-147.

Rosenberger, W. F., Lachin, J. L. (2002). Randomization in Clinical Trials: Theory and Practice. New York: Wiley.

Rosenberger, W. F., Seshaiyer, P. (1997). Adaptive survival trials. Journal of Biopharmaceutical Statistics, 7: 617-624.

Rosenberger, W. F., Stallard, N., Ivanova, A., Harper, C. N., Ricks, M. L. (2001). Optimal adaptive designs for binary response trials, Biometrics, 57, 909-913.

Rosenberger, W. F., Sverdlov, O., Hu, F. (2012). Adaptive randomization for clinical trials. Journal of Biopharmaceutical Statistics, 22: 719-736.

Sun, R., Cheung, S. H., Zhang, L.-X. (2007). A generalized drop-the-loser rule for multi-treatment clinical trials. Journal of Statistical Planning and Inference, 137: 2011-2023.

Sverdlov, O., Rosenberger, W. F. (2013a). On recent advances in optimal allocation designs in clinical trials. Journal of Statistical Theory and Practice, 7, 753-773.

Sverdlov, O., Rosenberger, W. F. (2013b). Randomization in clinical trials: can we eliminate bias? Clinical Investigation, 3(1), 37-47.

Sverdlov, O., Ryeznik, Y., Wong, W. K. (2012). Doubly adaptive biased coin designs for balancing competing objectives in time-to-event trials. Statistics and Its Interface, 5: 401-413.

Sverdlov, O., Ryeznik, Y., Wong, W. K. (2014). Efficient and ethical response-adaptive randomization designs for multi-arm clinical trials with Weibull time-to-event outcomes. Journal of Biopharmaceutical Statistics, 24(4):732-754.

Sverdlov, O., Tymofyeyev, Y., Wong, W. K. (2011). Optimal response-adaptive randomized designs for multi-armed survival trials. Statistics in Medicine, 30: 2890-2910.

Thall, P. F., Wathen, J. K. (2007). Practical Bayesian adaptive randomization in clinical trials. European Journal of Cancer, 43: 860-867.

Thompson, W. R. (1933). On the likelihood that one unknown probability exceeds another in the view of the evidence of the two samples. Biometrika, 25: 275-294.

Tymofyeyev, Y., Rosenberger, W. F., Hu, F. (2007). Implementing optimal allocation in sequential binary response experiments. Journal of the American Statistical Association, 102: 224-234.

Wei, L. J. (1979). The generalized Pólya's urn design for sequential medical trials.Annals of Statistics, 7: 291-296.

Wei, L. J., Durham, S. (1978). The randomized play-the-winner rule in medical trials. Journal of the American Statistical Association, 73: 840-843.

Wong, W. K., Zhu, W. (2008). Optimum treatment allocation rules under a variance heterogeneity model. Statistics in Medicine, 27: 4581-4595.

Zelen, M. (1969). Play the winner rule and the controlled clinical trial. Journal of the American Statistical Association, 64: 131-146.

Zhang, L., Rosenberger, W. F. (2006). Response-adaptive randomization for clinical trials with continuous outcomes. Biometrics, 62(2):562-569. 
Zhang, L., Rosenberger, W. F. (2007). Response-adaptive randomization for survival trials: the parametric approach. Applied Statistics, 56(2): 153-165.

Zhang, L.-X. (2012). The Gaussian approximation for generalized Friedman's urn model with heterogeneous and unbalanced updating. Science China-Mathematics, 55(11): 2379-2404.

Zhang, L.-X. (2014). Central limit theorems for a recursive stochastic algorithm with applications to adaptive designs. Manuscript.

Zhang, L.-X., Chan, W. S., Cheung, S. H., Hu, F. (2007). A generalized drop-the-loser urn for clinical trials with delayed responses.Statistica Sinica, 17: 387-409.

Zhang, L.-X. and Hu, F. (2009). The Gaussian approximation for multi-color generalized Friedman's urn model. Science in China, Series A, 52(6): 1305-1326.

Zhang, L.-X., Hu, F., Cheung, S. H. (2006). Asymptotic theorems of sequential estimation-adjusted urn models. Annals of Applied Probability, 16:340-369.

Zhang, L.-X., Hu, F., Cheung, S. H., Chan, W. S. (2011). Immigrated urn models-theoretical properties and applications. The Annals of Statistics, 39(1): 643-671.

Zhang, L.-X., Hu, F., Cheung, S.H. and Chan, W.S. (2014a). Asymptotic properties of multi-color randomly reinforced Pólya urns. Advances in Applied Probability, 46: 585-602.

Zhang, L.-X., Hu, F., Cheung, S. H., Chan, W. S. (2014b). Multiple-treatment efficient randomizedadaptive design with minimum selection bias. Manuscript.

Zhu, H., Hu F. (2009). Implementing optimal allocation for sequential continuous responses with multiple treatments. Journal of Statistical Planning and Inference, 139: 2420-2430. 